arXiv:0705.3540

SNUST-070501

\title{
M2-brane Probe Dynamics and Toric Duality
}

\author{
Seok Kim ${ }^{1}$, Sangmin Lee ${ }^{2}$, Sungjay Lee ${ }^{2}$ and Jaemo Park ${ }^{3,4}$ \\ ${ }^{1}$ School of Physics, Korea Institute for Advanced Study, Seoul 130-722, Korea \\ ${ }^{2}$ School of Physics and Astronomy, Seoul National University, Seoul 151-747, Korea \\ ${ }^{3}$ Department of Physics, Postech, Pohang 790-784, Korea \\ ${ }^{4}$ Postech Center for Theoretical Physics (PCTP), Pohang, 790-784, Korea
}

\begin{abstract}
We study the dynamics of a single M2-brane probing toric Calabi-Yau four-fold singularity in the context of the recently proposed M-theory crystal model of $\mathrm{AdS}_{4} / \mathrm{CFT}_{3}$ dual pairs. We obtain an effective abelian gauge theory in which the charges of the matter fields are given by the intersection numbers between loops and faces in the crystal. We argue that the probe theory captures certain aspects of the $\mathrm{CFT}_{3}$ even though the true M2-brane CFT is unlikely to be a usual gauge theory. In particular, the moduli space of vacua of the gauge theory coincides precisely with the Calabi-Yau singularity. Toric duality, partial resolution, and a possibility of new RG flows are also discussed.
\end{abstract}




\section{Introduction}

Throughout the development of the AdS/CFT correspondence [1] for the past decade, the $\mathrm{AdS}_{4} / \mathrm{CFT}_{3}$ cases in M-theory has remained much less understood than the $\mathrm{AdS}_{5} / \mathrm{CFT}_{4}$ cases in IIB string theory. The lack of a perturbative description of M-theory and its M2/M5-brane world-volume theories poses a major obstacle.

When supergravity serves as a good approximation to the full theory, M-theory on $\mathrm{AdS}_{4}$ is no more difficult than IIB string theory on $\mathrm{AdS}_{5}$. The difficulty still remains on the CFT side. While the $\mathrm{CFT}_{4}$ can be derived by quantizing open strings on the D3-branes probing a conical singularity, no systematic method is available to derive the world-volume theory of M2-branes near a singularity. In early works on $\mathrm{AdS}_{4} / \mathrm{CFT}_{3}$, proposals for the $\mathrm{CFT}_{3}$ were made based on global symmetries and analogy with quiver gauge theories on D-branes; see, for example, $[2,3,4,5,6]$. None of the proposals was systematic enough to be applied to less symmetric geometries such as the infinite families $Y^{p, q}[7,8,9]$.

For M2-branes probing toric Calabi-Yau four-fold $\left(\mathrm{CY}_{4}\right)$ cones, a first step toward constructing the $\mathrm{CFT}_{3}$ was taken in $[10,11]$. It is an M-theory generalization of the brane-tiling model $[12,13$, $14,15,16]$, which has proven extremely successful in studying the $\mathrm{CY}_{3} / \mathrm{CFT}_{4}$ counterpart. As the information on the $\mathrm{CFT}_{3}$ is encoded in a three dimensional periodic lattice, the model was named the M-theory crystal model. Following [11], we will distinguish the $\mathrm{D} 3 / \mathrm{CY}_{3}$ model from the $\mathrm{M} 2 / \mathrm{CY}_{4}$ model by referring to the former as the tiling model and the latter as the crystal model.

By construction, the tiling/crystal models share many features, for example,

\begin{tabular}{rll} 
Tiling/Crystal & & $\mathrm{CFT}_{4} / \mathrm{CFT}_{3}$ \\
\hline edges (bonds) & $\leftrightarrow$ & matter fields \\
vertices (atoms) & $\leftrightarrow$ & super-potential terms \\
faces (tiles) & $\leftrightarrow$ & gauge groups
\end{tabular}

This is by now a well established dictionary in the tiling model. As for the crystal model, the first two items were verified in $[10,11]$, but the third one posed a puzzle. The graph of the tiling model splits the unit cell $\left(T^{2}\right)$ into disjoint faces (tiles), and the D5/NS5-brane interpretation [13, 17] of 
the model explains why gauge groups are assigned to the faces. In contrast, the graph of the crystal model does not divide the unit cell $\left(T^{3}\right)$ into disjoint cells. At first sight, the crystal model does not seem to assign any role to faces and cells.

There is another related problem. In the crystal model, the fundamental fields of the $\mathrm{CFT}_{3}$ are interpreted as open M2-brane discs ending on M5-branes, and the "gauge-invariant" operators as closed spherical M2-branes formed by gluing the M2-discs. It requires a new algebraic structure beyond the usual matrix product. So we are tempted to conclude that the $\mathrm{CFT}_{3}$ cannot be written down until the full "non-abelian" M5-brane theory is constructed, which is a notoriously difficult subject on its own.

If the "non-abelian" nature of M-branes is the major obstacle to further progress, we may ask whether the problem can be simplified by considering a single $\mathrm{M} 2$-brane probe. In the $\mathrm{D} 3 / \mathrm{CY}_{3}$ case, the abelian gauge theory for a single D3-brane probe proved useful in the early stages of the development $[18,19,20]$ as well as in the discovery of the tiling model. In general, the probe theory does not exhibit the dynamics of the full non-abelian theory. For example, the super-potential often vanish in the abelian theory while it plays an essential role in the non-abelian theory. On the other hand, the probe theory does encode some important features of the full theory such as the multiplicity of matter super-fields and the moduli space of vacua which coincide with the $\mathrm{CY}_{3}$ singularity.

Bearing in mind both the usefulness and the limitation of the D3-brane probe theory, in the present paper, we explore the probe theory of an M2-brane in the context of the crystal model. The crystal model maps the M2-brane probe to an M5-brane. The information on the toric $\mathrm{CY}_{4}$ geometry is reflected in the shape of the M5-brane in the "internal" space. We take the world-volume theory of the M5-brane to be a free CFT with a single tensor multiplet of $(2,0)$ supersymmetry in six dimension. Once we "compactify" the M5-brane theory along the internal space, we obtain an effective abelian gauge theory in $(2+1)$-dimensions with $\mathcal{N}=2$ supersymmetry. As the open M2-brane discs are charged under the self-dual tensor field on the M5-brane, they indeed become charged matter fields in the probe theory. Moreover, the gauge groups can be attributed to faces 
of the crystal as in the dictionary above. The charges of the matter fields are then given by the intersection numbers between loops and faces in the crystal.

Some cautionary remarks are in order. There is no reason to expect that the M2-brane theory is a gauge theory. In fact, the crystal model points strongly to the contrary. The abelian gauge theory under discussion should be regarded, at best, as an approximate description of the true theory, to the same extent as the M5-brane theory is an abelian tensor gauge theory. Physical consequences derived from the probe theory may or may not survive in the full "non-abelian" theory. In either case, we find it reasonable to expect the probe theory to be a useful guide toward the construction of the "non-abelian" theory.

Partial resolution, which we study in section 6, is a good example to illustrate the point. We will show that the M2-brane probe theory admits partial Higgsing which translates to partial resolution of the $\mathrm{CY}_{4}$ singularity. An important result here is that this theory does not allow all possible resolution that a D3-brane probe does. Suppose a dual explanation of this difference can be found in terms of the difference between IIB supergravity and M supergravity (a question we hope to address in a future work). Then, it would impose a non-trival constraint on any future candidates for the "non-abelian" $\mathrm{CFT}_{3}$. Our discussion in section 7 on possible RG flows could serve similar purpose.

This paper is organized as follows. We begin with a brief review of the crystal model $[10,11]$ in section 2. In section 3, we explain how to identify the $U(1)$ gauge groups and compute the charges from the crystal model. We also explain how the charge assignment rule can be derived from the world-volume theory of a single M5-brane. In section 4, we show that the moduli space of vacua coincides with the toric $\mathrm{CY}_{4}$, thereby confirming the validity of the theory. In section 5, we show that two different gauge theories can correspond to the same $\mathrm{CY}_{4}$ in a way similar to the toric duality of [20]. Toric duality of non-abelian $\mathrm{CFT}_{4}$ is known to be a Seiberg duality. The meaning of toric duality of "non-abelian" $\mathrm{CFT}_{3}$ is an interesting open question. In section 6, we study partial Higgsing of a theory to obtain another one, thereby partially resolving the $\mathrm{CY}_{4}$ singularity, following similar consideration of the abelian $\mathrm{CFT}_{4}$ [20]. In section 7, we note that the 
abelian theory allows a "massive deformation" analogous to the Klebanov-Witten flow [21] from an orbifold CFT to the conifold CFT. We conclude with some discussions in section 8 .

\section{M-theory Crystal Model: a brief review}

We give a brief review of the M-theory crystal model $[10,11]$. The crystal model relates a toric $\mathrm{CY}_{4}$ to a three-dimensional periodic graph (a crystal) which encodes informations on the $\mathrm{CFT}_{3}$. As in $[20,15,11]$, the maps between a crystal and the corresponding toric diagram will be called the forward and inverse algorithms; see Figure 1.
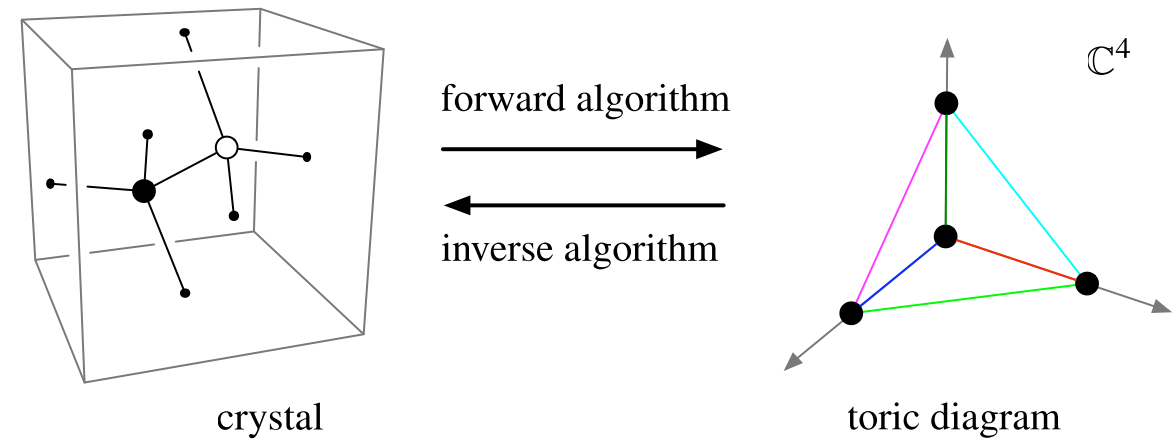

toric diagram

Figure 1: Crystal vs. Toric diagram.

The toric diagram forms a convex polyhedron in $\mathbb{Z}^{3} \subset \mathbb{Z}^{4}$. The reduction from $\mathbb{Z}^{4}$ to $\mathbb{Z}^{3}$ is a consequence of the CY condition. See, for example, $[22,23]$ for more information on toric geometry in this context.

The crystal model follows from a T-duality of M-theory. We take the T-duality transformation along $T^{3} \subset T^{4}$ in alignment with the projection $\mathbb{Z}^{4} \rightarrow \mathbb{Z}^{3}$. This corresponds to $x^{6,7,8}$ directions in Table 1 below. By T-duality, we mean the element $t$ in the $S L(2, \mathbb{Z}) \times S L(3, \mathbb{Z})$ duality group which acts as $t: \tau \equiv C_{(3)}+i \sqrt{g_{T^{3}}} \rightarrow-1 / \tau$. The stack of $N$ M2-branes turns into a stack of $N$ M5-branes wrapping the dual $T^{3}$. We call them the $T$-branes. The degenerating circle fibers turn into another M5-brane extended along the $(2+1) \mathrm{d}$ world-volume and a non-trivial 3-manifold $\Sigma$ in $\mathbb{R}^{3} \times T^{3}$. We call it the $\Sigma$-brane. Preservation of supersymmetry requires that the $\Sigma$-brane wrap 
a special Lagrangian submanifold of $\mathbb{R}^{3} \times T^{3}=\left(\mathbb{C}^{*}\right)^{3}$, and that it is locally a plane in $\mathbb{R}^{3}$ and a 1-cycle in $T^{3}$. The result is summarized in Table 1 .

\begin{tabular}{|c|c|c|c|c|c|c|c|c|c|}
\hline & 0 & 1 & 2 & $\begin{array}{lll}3 & 4 & 5\end{array}$ & 56 & 7 & 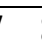 & 3 & \\
\hline M5 & 0 & $\overline{0}$ & $\overline{0}$ & & & O & & D & \\
\hline M5 & ○ & o & o & & $\Sigma$ & & & & \\
\hline
\end{tabular}

Table 1: The brane configuration for the $\mathrm{CFT}_{3}$. Away from the origin of $\mathbb{R}^{3}(345)$, the special Lagrangian manifold $\Sigma$ is locally a product of a 2-plane in $\mathbb{R}^{3}(345)$ and a 1 -cycle in $T^{3}(678)$.

The crystal graph is the intersection locus between the $T$-branes and the $\Sigma$-brane projected onto the $T^{3}$ (the alga projection). It was shown in [10] that the matter fields of the $\mathrm{CFT}_{3}$ are the M2discs whose boundaries encircle the bonds of the crystal, hence the first item of the dictionary we discussed in the introduction. The derivation of the M2-disc picture made use of the fact that the $T$ branes and the $\Sigma$-brane can be merged into a single, smooth, brane configuration. This observation will play a crucial role when we later discuss the gauge groups. It was also argued in $[10,11]$ that the atoms (vertices) of the crystal give the super-potential terms. As an evidence, it was shown to reproduce the BPS spectrum of meson operators of the $\mathrm{CFT}_{3}$.

In principle, the forward algorithm consists of two simple steps: (a) reading off the $\mathrm{CFT}_{3}$ (b) showing that its moduli space of vacua gives the toric $\mathrm{CY}_{4}$. These steps were not directly verified in $[10,11]$ since the information on the gauge group was missing. Instead, it was shown that the "fast" forward algorithm $[12,13]$ of the tiling model can be applied without any modification. The fast forward algorithm is based on the concept of perfect matchings. A perfect matching is a subset of bonds of the crystal, such that every atom of the crystal is an end-point of precisely one such bond. The bonds in each perfect matching carry an orientation. We choose to orient the arrows to go from a white atom to a black one. Perfect matchings have several nice properties. In particular,

1. Each perfect matching can be located in the toric diagram. The relative coordinate in the toric diagram between two perfect matchings $p_{\alpha}$ and $p_{\beta}$ is given by the homology charge of $\left(p_{\alpha}-p_{\beta}\right)$ regarded as a one-cycle in $T^{3}$.

2. The perfect matchings solve the 'abelian' version of the F-term condition for the chiral fields 
$X_{i}$ associated to the bonds, if we set

$$
X_{i}=\prod_{\alpha} p_{\alpha}^{\left\langle X_{i}, p_{\alpha}\right\rangle},
$$

where $\left\langle X_{i}, p_{\alpha}\right\rangle$ equals 1 if $p_{\alpha}$ contains the bond $X_{i}$ and 0 otherwise.

The abelian gauge theory of the present paper fills the gap by showing that the "slow" forward algorithm also works. The perfect matchings will continue to be a useful device.

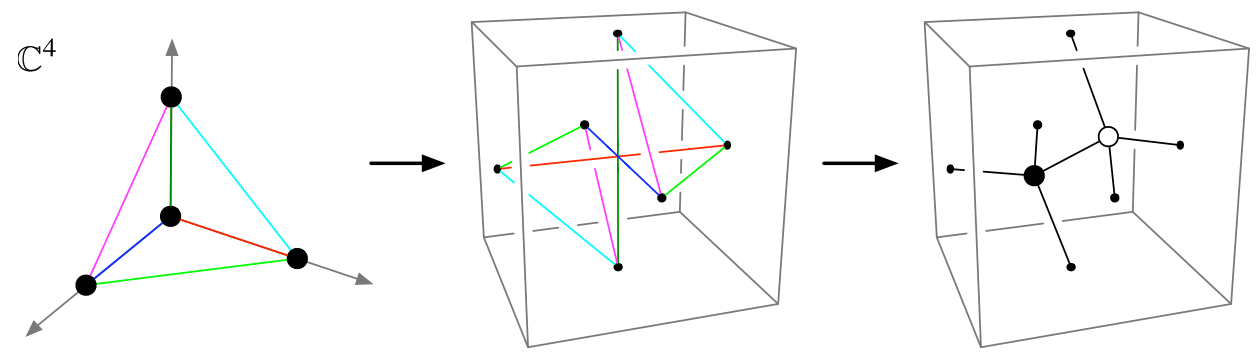

Figure 2: Inverse algorithm for $\mathbb{C}^{4}$, reproduced from [11].

The inverse algorithm is not as well established as the forward algorithm. Roughly speaking, one draws a 1-cycle in the $T^{3}$ for each edge of the toric diagram and then merge the 1-cycles to make up the crystal. For simple examples with high degree of symmetry, the procedure is unambiguous. Even in the general case, the result can be checked by using the forward algorithm. It is certainly desirable to understand the inverse algorithm better by finding an explicit expression for the special Lagrangian manifold $\Sigma$.

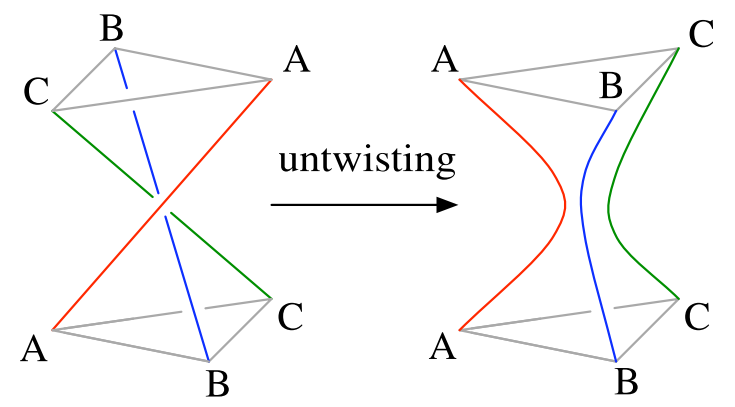

Figure 3: Untwisting from alga to amoeba, reproduced from [11]. 
It is useful to consider the projection of the crystal onto $\Sigma$ (the amoeba projection). We can think of the amoeba as a 3-manifold with some defects. As we shrink the 2-fans along the radial direction, the "points at infinity" form a locally one dimensional defect. The 1-cycles paired with the 2-fans are localized along the defects. Globally, the defect is isomorphic to the dual toric diagram. When the toric diagram has no internal points, the amoeba has the topology of a threesphere apart from the defect.

It is possible to obtain the amoeba from the alga and vice versa using the untwisting procedure. The untwisting flips the orientation of the space transverse to the bond of the crystal See Figure 3. We apply the untwisting map to the alga of $\mathbb{C}^{4}$, we obtain the amoeba depicted in Figure 4 . Note that the dual toric diagram is a tetrahedron as expected. The 1-cycles are localized along the dual toric diagram as they should be. Applying the untwisting map to $C\left(Q^{1,1,1}\right)$ yields a similar result, with the dual toric diagram being a cube.
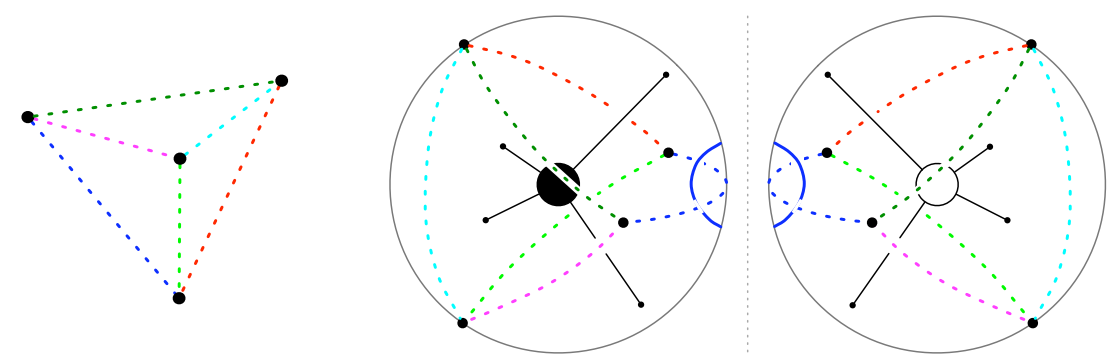

Figure 4: Dual toric diagram and amoeba diagram for $\mathbb{C}^{4}$, reproduced from [11]. We represent a three-sphere as the union of two balls with the surfaces identified. The dotted lines on the balls denote the defects.

We close this section with Figure 5 which contains the toric diagrams of $\mathrm{CY}_{4}$ we consider in this paper. The corresponding crystals will be shown in later sections. 
(a) $\mathbb{C}^{4}$

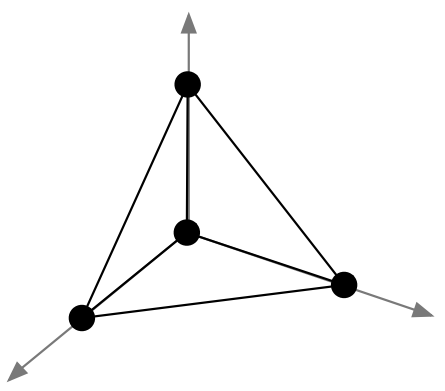

(b) $\mathbb{C}^{2} / \mathbb{Z}_{2} \times \mathbb{C}^{2}$

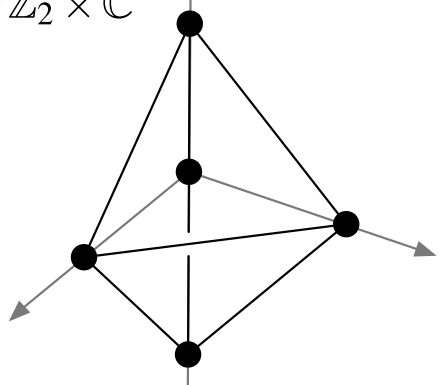

(c)

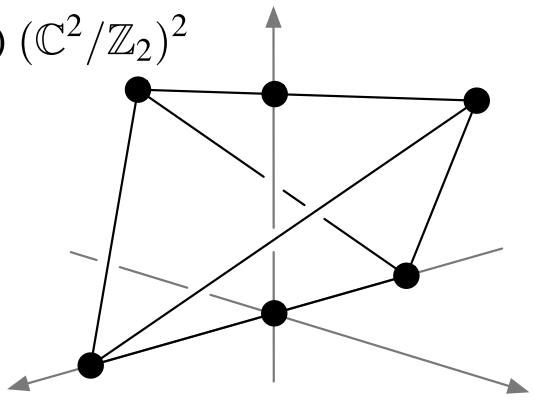

(d) $\left(\mathbb{C}^{3} / \mathbb{Z}_{2} \times \mathbb{Z}_{2}\right) \uparrow \times \mathbb{C}$

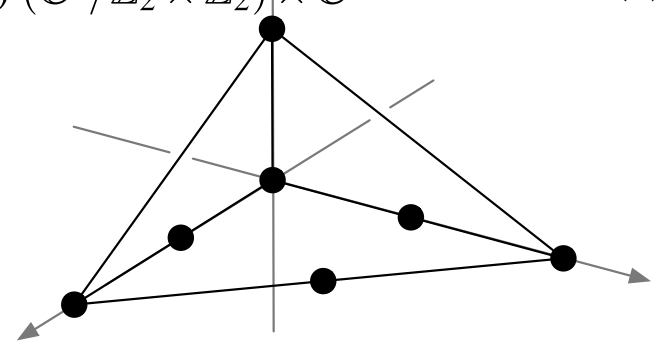

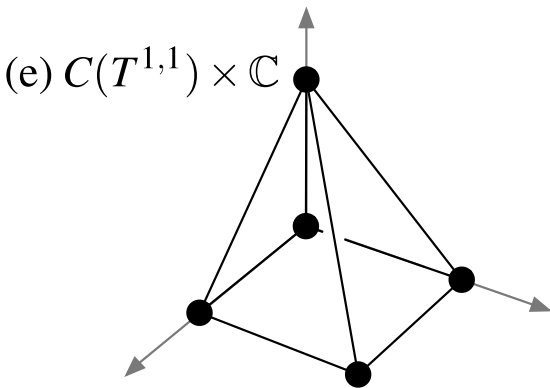

(f) $C\left(Q^{1,1,1}\right)$

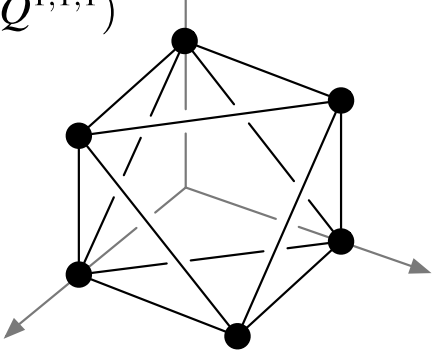

(g) $d P_{3} \times \mathbb{C}$

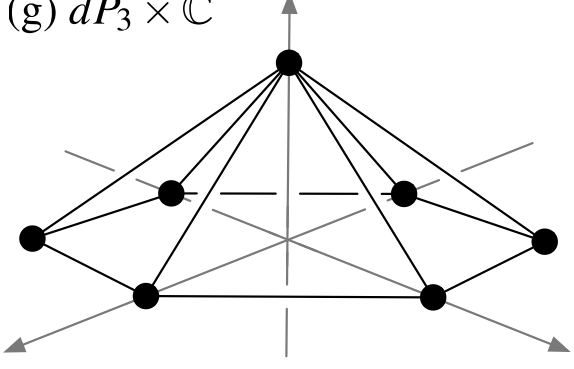

(h) $D_{3}$

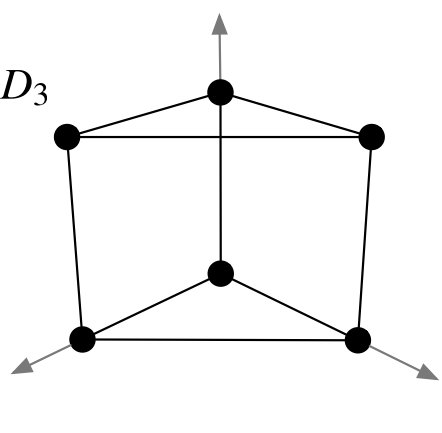

Figure 5: Toric diagrams of the $\mathrm{CY}_{4}$ considered in this paper. 


\section{Abelian Gauge Theory}

\subsection{Charge assignment rule}

The main feature of the abelian gauge theory associated to the crystal model is that the $U(1)$ gauge groups are attributed to faces of the crystal. Recall that a matter field in the crystal model is represented by an M2-brane disc whose boundary loop is localized along a bond of the crystal. Then, the charges of the matter fields are simply the intersection number between the "matter loops" and the "charge faces." Figure 6 gives a pictorial description of the charge assignment rule. In the figure, we fixed the orientation of the matter loops by imagining arrows from white atoms to black ones and applying the right-hand rule. This ensures that the M2-discs surrounding an atom can form an orientable two-sphere.
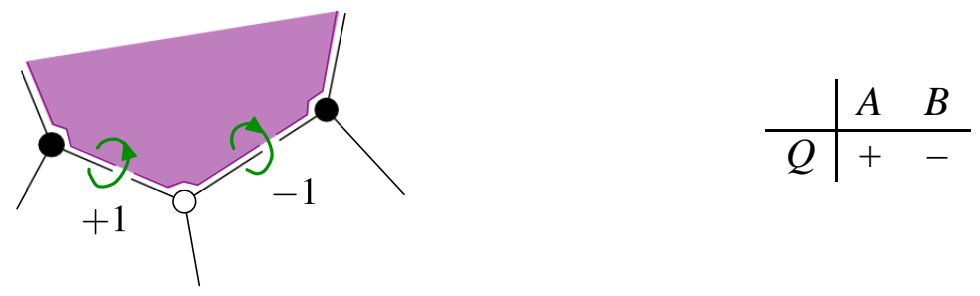

Figure 6: Charges as intersection numbers between the "matter loops" and the "charge faces."

Although this is quite reminiscent of the charge assignment in the tiling model, we should emphasize the difference between the two models. In the D5/NS5-brane picture of the tiling model, it is very clear how the disjoint tiles of D5-branes give rise to gauge groups for the matter fields that are open strings connecting the tiles. The role of faces in the crystal model is not very clear at first sight. In addition, the number of independent gauge groups is not so easy to count as in the tiling model.

We will shortly explain the M-theory origin of the charge assignment as well as how to count the number of gauge groups. Before doing so, we give a concrete example of the charge assignment in Figure 7. It has two independent charges. This example is especially easy to visualize because the faces lie on planes, which is not true in general. 


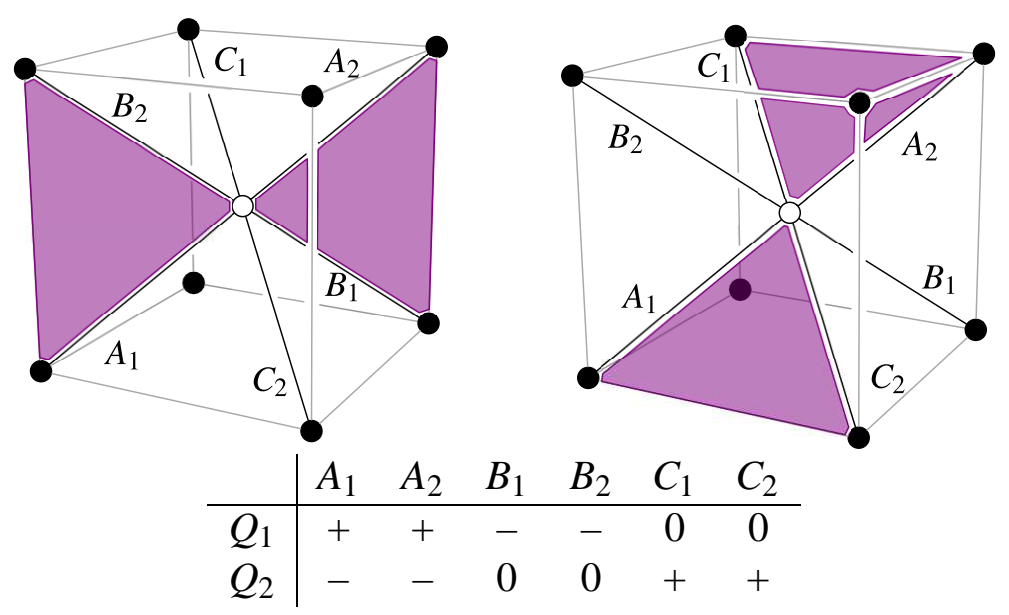

Figure 7: Two independent faces for $C\left(Q^{1,1,1}\right)$.

\subsection{M-theory origin}

As we reviewed in section 2, the crystal model comes from two types of M5-branes: the $T$-brane and the $\Sigma$-brane. An important step, which was used already in identifying the matter fields as open M2-branes, is that the two M5-branes can merge into a single M5-brane of a complicated topology. Let us call it $\mathcal{M}$. This M5-brane carries on its world-volume the "self-dual" two-form field $B$. The abelian gauge fields of the crystal model come from the Kaluza-Klein reduction of the $B$-field along the internal manifold $\mathcal{M}$.

Open M2 branes ending on an M5-brane couples to the 'self-dual' two-form field $B$ as

$$
S_{M 2} \sim \int_{\partial D} B
$$

When the M5-brane is extended over $\mathbb{R}^{1,2} \times \mathcal{M}$, we can use harmonic one-forms on $\mathcal{M}$ to decompose $B$ as

$$
B(x, y)=A^{a}(x) \wedge \omega_{a}(y)+\cdots,
$$

where the omitted terms are required to ensure the self-duality condition $d B=* d B$.

An M2-disc whose boundary is a 1-cycle $C_{i}$ in $\mathcal{M}$ become a particle in (2+1)-dimension with 
a coupling to the gauge field,

$$
S_{i} \sim Q_{i a} \int A^{a}
$$

The charge is easy to read off

$$
Q_{i a}=\int_{C_{i}} \omega_{a}=\sharp\left(C_{i}, S_{a}\right) .
$$

In the last step, we replaced the integral of $\omega_{a}$ over $C_{i}$ by the intersection number between $C_{i}$ and the two-cycle $S_{a}$ which is Poincaré dual to $w_{a}$.
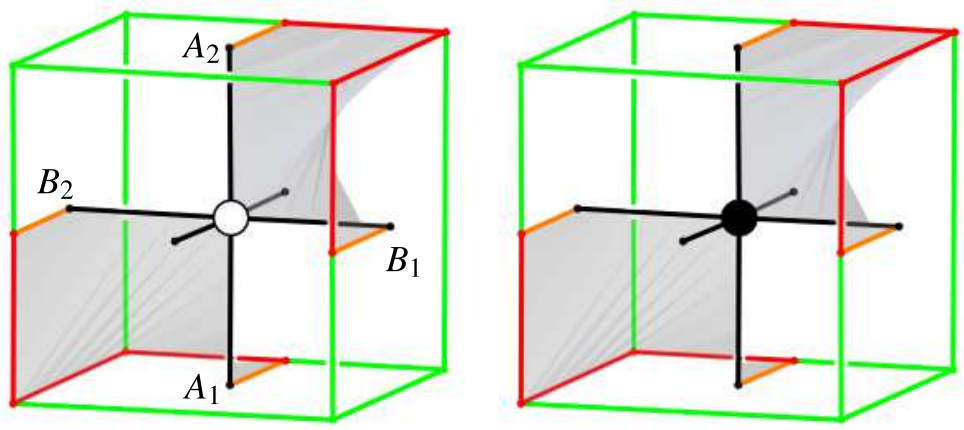

Figure 8: A 2-cycle in the amoeba diagram of $C\left(Q^{1,1,1}\right)$. The union of the two solid cubes with the surfaces identified represent a three-sphere. The edges of the cube (green line) denote the dual toric diagram at asymptotic infinity.

It remains to show that faces of the crystal define 2-cycles of $\mathcal{M}$. When the $T$-brane and $\Sigma$-brane merge to form $\mathcal{M}$, the bonds of the crystal serve as the "throat" connecting the two components. So, the faces should be carried over to the $\Sigma$ side. Recall that the transition from the crystal to the amoeba involves the untwisting process. It means that the tangent plane to the face go through a $180^{\circ}$ rotation as it moves from an atom to its neighbor along a bond.

In the amoeba diagram, the bonds of the crystal and the dual toric diagram are linked nontrivially. So, it is impossible for the extended face to form a compact 2-cycle of $\mathcal{M}$. The only alternative is that it runs off to infinity to form a non-compact 2-cycle. An example of a such a 2-cycle is depicted in Figure 8. In the figure, the face emanates from the bonds $\left(A_{1}-B_{2}-A_{2}-B_{1}\right)$ 
where $A_{i}, B_{i}$ are define in Figure 7. It is twisted in accordance with the untwisting process, and it runs off to the dual toric diagram at infinity. The red segments of the dual toric diagram denote the asymptotic boundary of the face.

We have checked for all examples considered in this paper that any face of the crystal can be extended to the amoeba diagram and sent to asymptotic infinity. We take it as a strong evidence that it works for any crystal, but we have not found a general proof yet.

\subsection{Examples}

To illustrate the charge assignment rule, we present a few more examples in the following set of figures along with tables summarizing the charges. The faces are not drawn in the figures explicitly, but the charge table can be used to figure out the faces for each charge.

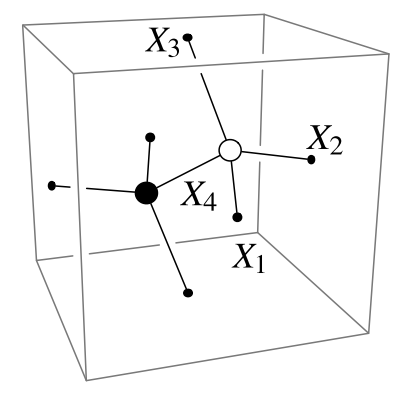

All $X_{i}$ 's are neutral and $W=0$.

Figure 9: $\mathbb{C}^{4}$

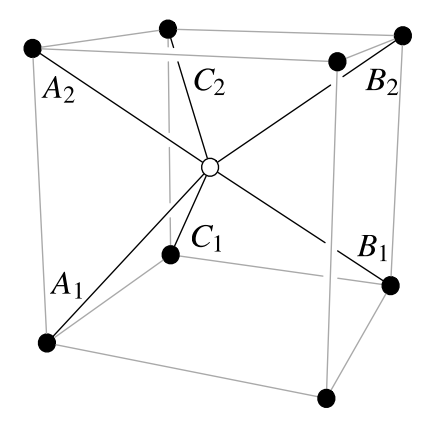

\begin{tabular}{c|cccccc} 
& $A_{1}$ & $A_{2}$ & $B_{1}$ & $B_{2}$ & $C_{1}$ & $C_{2}$ \\
\hline$Q_{1}$ & + & - & - & + & 0 & 0 \\
$Q_{2}$ & - & + & 0 & 0 & + & - \\
$W=0$. & & & &
\end{tabular}

Figure 10: $D_{3}$

An astute reader may have noticed that the charge matrix of $C\left(Q^{1,1,1}\right)$ and that of $d P_{3} \times \mathbb{C}$ are the same except that $C\left(Q^{1,1,1}\right)$ does not have the $\phi_{1,2}$ fields that $d P_{3} \times \mathbb{C}$ has. Similarly, $D_{3}$ and 


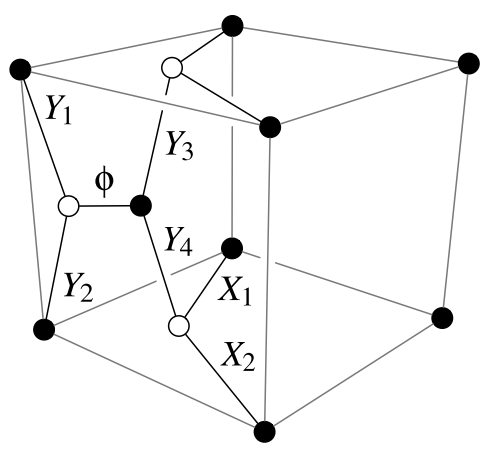

$$
\begin{array}{c|ccccccc} 
& \phi & X_{1} & X_{2} & Y_{1} & Y_{2} & Y_{3} & Y_{4} \\
\hline Q & 0 & 0 & 0 & + & - & + & - \\
W= & \phi\left(Y_{1} Y_{2}-Y_{3} Y_{4}\right)-X_{1} X_{2}\left(Y_{1} Y_{2}-Y_{3} Y_{4}\right) .
\end{array}
$$

Figure 11: $\mathbb{C}^{2} / \mathbb{Z}_{2} \times \mathbb{C}^{2}$

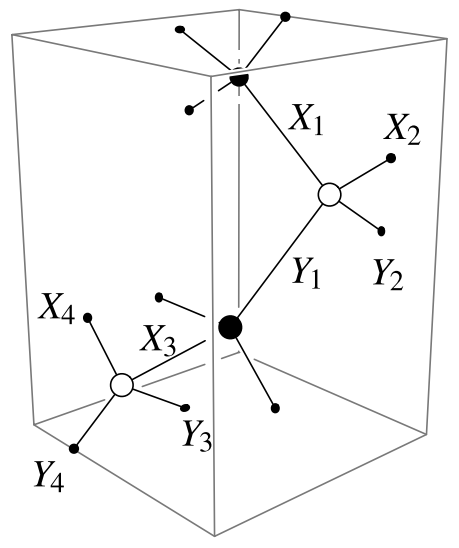

\begin{tabular}{c|cccccccc} 
& $X_{1}$ & $X_{2}$ & $X_{3}$ & $X_{4}$ & $Y_{1}$ & $Y_{2}$ & $Y_{3}$ & $Y_{4}$ \\
\hline$Q_{1}$ & - & + & - & + & 0 & 0 & 0 & 0 \\
$Q_{2}$ & 0 & 0 & 0 & 0 & + & - & + & -
\end{tabular}

$W=X_{1} X_{2} Y_{1} Y_{2}-X_{3} X_{4} Y_{1} Y_{2}+X_{3} X_{4} Y_{3} Y_{4}-X_{1} X_{2} Y_{3} Y_{4}$.

Figure 12: $\left(\mathbb{C}^{2} / \mathbb{Z}_{2}\right)^{2}$

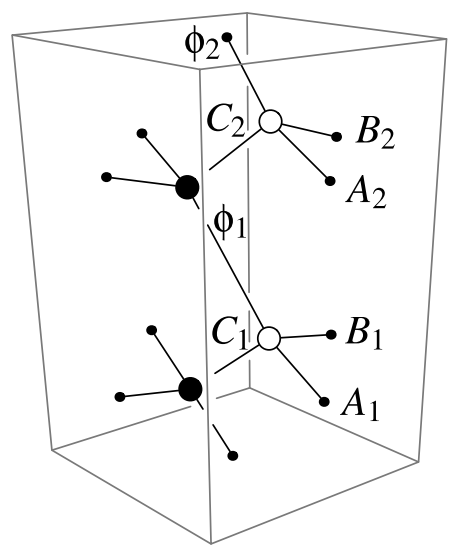

$$
\begin{array}{c|cccccccc} 
& \phi_{1} & \phi_{2} & A_{1} & A_{2} & B_{1} & B_{2} & C_{1} & C_{2} \\
\hline Q_{1} & 0 & 0 & + & - & - & + & 0 & 0 \\
Q_{2} & 0 & 0 & - & + & 0 & 0 & + & - \\
W= & \phi_{1}\left(A_{1} B_{1} C_{1}-A_{2} B_{2} C_{2}\right)-\phi_{2}\left(A_{1} B_{1} C_{1}-A_{2} B_{2} C_{2}\right) .
\end{array}
$$

Figure 13: $\mathbb{C}^{3} /\left(\mathbb{Z}_{2} \times \mathbb{Z}_{2}\right) \times \mathbb{C}$ 


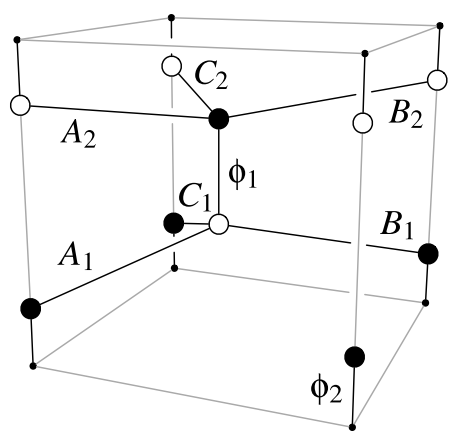

\begin{tabular}{c|cccccccc} 
& $\phi_{1}$ & $\phi_{2}$ & $A_{1}$ & $A_{2}$ & $B_{1}$ & $B_{2}$ & $C_{1}$ & $C_{2}$ \\
\hline$Q_{1}$ & 0 & 0 & + & + & - & - & 0 & 0 \\
$Q_{2}$ & 0 & 0 & - & - & 0 & 0 & + & + \\
$W=\phi_{1}\left(A_{1} B_{1} C_{1}-A_{2} B_{2} C_{2}\right)-\phi_{2}\left(A_{1} B_{1} C_{1}-A_{2} B_{2} C_{2}\right)$.
\end{tabular}

Figure 14: $d P_{3} \times \mathbb{C}$

$\mathbb{C}^{3} /\left(\mathbb{Z}_{2} \times \mathbb{Z}_{2}\right)$ have almost identical charge matrices. We will discuss their implications in section 7.

\section{Moduli Space of Vacua}

Given a toric $\mathrm{CY}_{4}$ and the associated crystal, it is natural to expect that the abelian theory defined in the previous section has a moduli space of vacua, $\mathcal{M}_{V}$, that coincides with the $\mathrm{CY}_{4}$. We now show that it is indeed the case. First, we present a few illustrative examples to familiarize the reader with the abelian theory and its $\mathcal{M}_{V}$. Then we give a general proof that $\operatorname{dim}\left(\mathcal{M}_{V}\right)=4$ in parallel with a similar proof in the tiling model. Finally, we make a comparison with the D-brane gauge theory in the $\mathcal{N}=4$ cases.

\subsection{Examples}

1. $C\left(Q^{1,1,1}\right)$

We have eight gauge invariant variables $z_{i j k}=A_{i} B_{j} C_{k}(i, j, k=1,2)$. Label them as

$$
\begin{aligned}
& z_{0}=A_{1} B_{1} C_{1}, z_{1}=A_{1} B_{1} C_{2}, z_{2}=A_{1} B_{2} C_{1}, z_{3}=A_{1} B_{2} C_{2}, \\
& z_{4}=A_{2} B_{1} C_{1}, z_{5}=A_{2} B_{1} C_{2}, \quad z_{6}=A_{2} B_{2} C_{1}, z_{7}=A_{2} B_{2} C_{2} .
\end{aligned}
$$


They satisfy the quadratic relations

$$
\begin{aligned}
& z_{0} z_{7}=z_{1} z_{6}=z_{2} z_{5}=z_{3} z_{4}, \\
& z_{0} z_{3}=z_{2} z_{1}, \quad z_{7} z_{4}=z_{5} z_{6}, \\
& z_{0} z_{5}=z_{1} z_{4}, \quad z_{7} z_{2}=z_{6} z_{3}, \\
& z_{0} z_{6}=z_{4} z_{2}, \quad z_{7} z_{1}=z_{3} z_{5} .
\end{aligned}
$$

As noted earlier in [2, 5], this is precisely the algebraic definition of $C\left(Q^{1,1,1}\right)$.

2. $\mathbb{C}^{2} / \mathbb{Z}_{2} \times \mathbb{C}^{2}$

In addition to the neutral fields, $\phi, X_{1}, X_{2}$, we have four gauge invariant variables: $w_{1}=Y_{1} Y_{2}$, $w_{2}=Y_{3} Y_{4}, u_{1}=Y_{1} Y_{4}, u_{2}=Y_{2} Y_{3}$. The F-term conditions demands that

$$
\phi=X_{1} X_{2}, \quad w_{1}=w_{2}(\equiv w)
$$

We note that $\left(X_{1}, X_{2}\right)$ span the $\mathbb{C}^{2}$ part and the algebraic relation, $u_{1} u_{2}=w^{2}$, describes $\mathbb{C}^{2} / \mathbb{Z}_{2}$.

3. $\left(\mathbb{C}^{2} / \mathbb{Z}_{2}\right)^{2}$

The $X$ fields and $Y$ fields decouple from each other. The F-term condition demands that $X_{1} X_{2}=X_{3} X_{4}(\equiv w)$. The gauge-invariant coordinates $u_{1}=X_{1} X_{4}$ and $u_{2}=X_{2} X_{3}$ satisfy $u_{1} u_{2}=$ $w^{2}$, which gives $\mathbb{C}^{2} / \mathbb{Z}_{2}$. Similarly, the $Y$ fields produce another factor of $\mathbb{C}^{2} / \mathbb{Z}_{2}$.

4. $\mathbb{C}^{3} /\left(\mathbb{Z}_{2} \times \mathbb{Z}_{2}\right) \times \mathbb{C}$

There are seven gauge invariant coordinates, $\phi_{1}, \phi_{2}, w_{1} \equiv A_{1} B_{1} C_{1}, w_{2} \equiv A_{2} B_{2} C_{2} z_{1} \equiv A_{1} A_{2}$, $z_{2} \equiv B_{1} B_{2}, z_{3} \equiv C_{1} C_{2}$, and two F-term conditions,

$$
\phi_{1}=\phi_{2}(\equiv \phi), \quad w_{1}=w_{2}(\equiv w) .
$$

Clearly, $\phi$ parameterizes the $\mathbb{C}$ factor and the algebraic relation, $z_{1} z_{2} z_{3}=w^{2}$, describes the orbifold $\mathbb{C}^{3} /\left(\mathbb{Z}_{2} \times \mathbb{Z}_{2}\right)$. 
5. $d P_{3} \times \mathbb{C}$

The gauge-invariant coordinates are $\phi_{1}, \phi_{2}$ and $z_{i j k}=A_{i} B_{j} C_{k}$. The F-term condition gives two linear constraints:

$$
\phi_{1}=\phi_{2}(\equiv \phi), \quad z_{111}=z_{222}(\equiv w)
$$

Label the remaining six $z_{i j k}$ as

$$
\begin{array}{ll}
s_{1}=A_{2} B_{1} C_{1}, & s_{2}=A_{1} B_{2} C_{2}, \\
t_{1}=A_{1} B_{2} C_{1}, & t_{2}=A_{2} B_{1} C_{2}, \\
u_{2}=A_{1} B_{1} C_{2}, & u_{2}=A_{2} B_{2} C_{1} .
\end{array}
$$

The gauge-invariant coordinates are subject to the quadratic relations,

$$
\begin{aligned}
& w^{2}=s_{1} s_{2}=t_{1} t_{2}=u_{1} u_{2}, \\
& w s_{1}=t_{2} u_{2}, \quad w s_{2}=t_{1} u_{1}, \\
& w t_{1}=u_{2} s_{2}, \quad w t_{2}=u_{1} s_{1}, \\
& w u_{1}=s_{2} t_{2}, \quad w u_{2}=s_{1} t_{1} .
\end{aligned}
$$

This coincides with the known algebraic definition of $d P_{3}$. As a check, we note that the character function of $d P_{3}$ [24],

$$
Z=\frac{1+4 t+t^{2}}{(1-t)^{3}}=1+7 t+19 t^{2}+O\left(t^{3}\right)
$$

requires that there be seven monomials of degree one and nineteen independent monomials 
of degree two. It is easy to see that we have the correct number of monomials.

$$
\begin{aligned}
\operatorname{deg} 1: & w, s_{i}, t_{i}, u_{i} \\
\operatorname{deg} 2: & w^{2} ; w s_{i}, w t_{i}, w u_{i} ; s_{i}^{2}, t_{i}^{2}, u_{i}^{2} ; \\
& s_{1} t_{2}, s_{2} t_{1}, t_{1} u_{2}, t_{2} u_{1}, u_{1} s_{2}, u_{2} s_{1} .
\end{aligned}
$$

All other monomials of degree two are redundant due to the quadratic relations.

\subsection{Dimension counting}

As a warm-up exercise, we first review how to count the dimension of $\mathcal{M}_{V}$ in the tiling model. Recall the well-known relations in the tiling model:

1. $\sharp($ gauge groups $)=\sharp($ faces $) \equiv f$.

2. $\sharp$ (bi-fundamentals $)=\sharp($ edges $) \equiv e$.

3. $\sharp($ super-potential terms $)=\sharp($ vertices $) \equiv v$.

To count $\operatorname{dim}\left(\mathcal{M}_{V}\right)$ of the abelian theory, we note that

1. $\sharp$ (F-term conditions $) \equiv w=v-2$.

The F-term conditions equate all the super-potential terms, leading to $v-1$ equations. One of them turns out to be redundant.

2. $\sharp($ D-term conditions $) \equiv q=f-1$.

The D-term conditions come from the $U(1)$ gauge groups. All matter fields are neutral under the diagonal $U(1)$, since they are all bi-fundamentals.

The dimension of $\mathcal{M}_{V}$ is the number of matter fields minus the number of constraints: $\operatorname{dim}\left(\mathcal{M}_{V}\right)=$ $e-w-q$. Combining the topological condition, $f-e+v=0$, with the relations mentioned above, we find

$$
\operatorname{dim}\left(\mathcal{M}_{V}\right)=e-(v-2)-(f-1)=3,
$$


in agreement with the fact that $\mathcal{M}_{V}$ coincides with the $\mathrm{CY}_{3}$.

A similar argument can be made for the abelian theory of the crystal model. The following relations continue to hold.

1. $\sharp($ bi-fundamentals $)=\sharp($ edges $) \equiv e$.

2. $\sharp($ super-potential terms $)=\sharp($ vertices $) \equiv v$.

3. $\sharp($ F-term conditions $) \equiv w=v-2$.

4. $\operatorname{dim}\left(\mathcal{M}_{V}\right)=e-w-q$, where $q$ is the number of D-term conditions.

Now, we introduce as many faces as we need to partition the $T^{3}$ into several cells, so that the topological condition $-c+f-e+v=0$ can be used. Here, $c$ and $f$ are the number of cells and faces, respectively. Note that, for a given crystal, $f-c=e-v$ is a topological invariant that does not depend on the details of the partition.

It is easy to see that $q$ depends only on the difference $f-c$. When a collection of faces surround a cell, the total charge vanishes identically, because the matter loops either penetrate the faces twice with opposite orientations or do not penetrate them at all. Therefore, we can remove faces while keeping $f-c$ fixed until we arrive at $c=1$. Let $\bar{f}$ be the number of faces in this configuration. By construction, $\bar{f}-1=f-c$.

The remaining $\bar{f}$ faces come in two distinct types. The first kind of faces live in the interior of the cell, so that they can be removed without changing $c=1$. All of them give independent $U(1)$ charges. The second kind of faces participate in forming the walls (2-cycles) surrounding the unit cell. The overall charge corresponding to the sum of all faces on the same wall vanishes, again because the loops either penetrate the faces twice or do not penetrate them at all. Since three independent walls are needed to make up a three-torus, the number of independent gauge groups gets reduced by three. Therefore, we have $q=\bar{f}-3=f-c-2$, which in turn implies

$$
\operatorname{dim}\left(\mathcal{M}_{V}\right)=e-(v-2)-(f-c-2)=4
$$

This is consistent with the fact that $\mathcal{M}_{V}$ coincides with the $\mathrm{CY}_{4}$. 


\subsection{Comparison with D-brane gauge theory}

It is widely believed that the world-volume theory of M2-branes in flat space-time can be obtained by taking the strongly coupled, infrared limit of the $\mathcal{N}=8$ super-Yang-Mills theory living on D2branes. The attempt to understand the M2-brane theory from the infrared limit of the D2-brane theory was extended to the $\mathcal{N}=4$, orbifold examples in [25]. We will now review the result of [25] in the abelian context and compare it with our new model.

The M2-branes probe the product of two singluar ALE (asymptotically locally euclidean) spaces. Regarding the $U(1)$-fiber in one of the ALE spaces as the M-theory circle, the theories can be considered as gauge theories on the D2-brane probe in type IIA theory with several D6 branes (and possibly O6 planes), transverse to $\mathbb{R}^{3}$.

To be concrete, we focus on the $\mathbb{C}^{2} / \mathbb{Z}_{n} \times \mathbb{C}^{2} / \mathbb{Z}_{k}$ examples. Compactifying on a circle in $\mathbb{C}^{2} / \mathbb{Z}_{k}$, we get $k$ D6-branes, transverse to $\mathbb{R}^{3}$, with the D2-brane probe. The D2-brane and all its mirror images under the $\mathbb{Z}_{n}$ orbifold action defines a $U(1)^{n}$ gauge theory. Each of the $n$ vector

multiplets has three scalars which represent the position in the transverse $\mathbb{R}^{3}$. The hyper-multiplets $X$ are bi-fundamentals connecting adjacent $U(1)$-factors of the gauge group [26]. In addition, there are other hyper-multiplets $\phi$ arising from the open string modes connecting the D2- and D6-branes, which are charged under only one of the $U(1)$ gauge groups.

There are two types of geometric moduli parameters we can add to the theory. Each D-term equation for the $X$ fields allows a Fayet-Iliopoulos (FI) parameter. Since all $X$ fields are neutral under the diagonal $U(1)_{D}$, we have $(n-1)$ independent FI parameters $\zeta_{i}$. They correspond to the moduli of the first ALE space, $\mathbb{C}^{2} / \mathbb{Z}_{n}$. One can also introduce the $k$ mass parameters, $m_{i}$, for the $\phi$ fields, which originate from the relative distance between D2- and D6-branes. The total mass $\sum_{i} m_{i}$ can be eliminated by shifting the origin of the Coulomb branch. The remaining $(k-1)$ mass parameters can be understood as the moduli of the second ALE space, $\mathbb{C}^{2} / \mathbb{Z}_{k}$.

In the $\mathcal{N}=2$ language, the $\mathcal{N}=4 \mathrm{D}$-term equations describing the Higgs branch of $X$ fields 
are given by

$$
\begin{aligned}
\left|X_{i, i+1}^{1}\right|^{2}-\left|X_{i+1, i}^{2}\right|^{2}-\left|X_{i-1, i}^{1}\right|^{2}+\left|X_{i, i-1}^{2}\right|^{2} & =\zeta_{i}^{D} \\
X_{i, i+1}^{1} X_{i+1, i}^{2}-X_{i-1, i}^{1} X_{i, i-1}^{2} & =\zeta_{i}^{F}
\end{aligned}
$$

where $i$ runs over $\{1, \cdots, n-1\}$. The real parameter $\zeta^{D}$ and complex parameter $\zeta^{F}$ together form a triplet of $\mathcal{N}=4$ FI parameters. The crystal model has only $\mathcal{N}=2$ manifest supersymmetry, and the meaning of $\zeta^{F}$ is not clear at present. The equations for the $\phi$ fields are

$$
\sum_{j=1}^{k-1}\left(\left|\phi^{1}{ }_{j}\right|^{2}-\left|\phi_{j}^{2}\right|^{2}\right)=0, \quad \sum_{j=1}^{k-1}\left(\phi_{j}^{1} \phi_{j}^{2}\right)=0 .
$$

Note that the equations for $\phi$ are decoupled from those for $X$ as well as the FI parameters.

Let us now examine $\mathcal{M}_{V}$ with both the mass and the FI parameters turned on. Since the fields $\phi$ become massive, their Higgs branch is lifted. Turning on the FI terms leads to breaking gauge symmetry down to the diagonal $U(1)_{D}$ under which only the fields $\phi$ are charged. Therefore, $\mathcal{M}_{V}$ is the product of the Higg branch, $\mathcal{M}_{H}$, for the $X$ fields described by the D-term equations (4.11) and the Coulomb branch, $\mathcal{M}_{C}$, for the $U(1)_{D}$ gauge theory with $k$ massive hyper-multiplets $\phi$.

As is well known, the D-terms equations (4.11) coincide with Kronheimer's hyper-Kähler quotient construction [27] of the first ALE space, $\mathbb{C}^{2} / \mathbb{Z}_{n}$, with the singularities resolved by the FI parameters. The Higgs branch, $\mathcal{M}_{H}$, does not receive any quantum corrections, and remains to be $\mathbb{C}^{2} / \mathbb{Z}_{n}$ even in the strong coupling regime.

The classical Coulomb branch for the $U(1)_{D}$ gauge theory is $\mathbb{R}^{3} \times S^{1}$. As in the $\mathcal{N}=8$ case, the periodic scalar is the Hodge dual of the $U(1)_{D}$ gauge field, and the radius of the circle goes to infinity in the IR limit. The quantum correction changes the Coulomb branch significantly [28], so that it describes the second ALE space $\mathbb{C}^{2} / \mathbb{Z}_{k}$ with the singularities resolved by the mass parameters. To summarize, the moduli space of vacua of the full theory is $\mathcal{M}_{V}=\mathcal{M}_{H} \times \mathcal{M}_{C}=$ $\mathbb{C}^{2} / \mathbb{Z}_{n} \times \mathbb{C}^{2} / \mathbb{Z}_{k}$, resolved by the FI and mass parameters.

If we now choose the M-theory circle in $\mathbb{C}^{2} / \mathbb{Z}_{n}$, instead of $\mathbb{C}^{2} / \mathbb{Z}_{k}$, then we have $n$ D6-branes 
with the D2-brane probe. Since the moduli space of vacua, $\widetilde{\mathcal{M}}_{V}$, of this dual theory should capture the same orbifold geometry, it must be that

$$
\mathfrak{M}_{H}=\widetilde{\mathcal{M}}_{C}, \quad \mathcal{M}_{C}=\widetilde{\mathscr{M}}_{H} .
$$

It is easy to confirm this from the gauge theory. In the dual theory, the first ALE space $\mathbb{C}^{2} / \mathbb{Z}_{n}$ is described by the Coulomb branch for $U(1)$ gauge theory with $n$ massive hyper-multiplets $\tilde{\phi}$ and the second $\mathbb{C}^{2} / \mathbb{Z}_{k}$ by the Higgs branch for $k$ hyper-multiplets $Y$. The duality also exchanges the mass parameters and the FI parameters. The key observation of [25] is that this is an example of the mirror symmetry of three dimensional gauge theories [29], and that M-theory "explains" why mirror symmetry holds in this case.

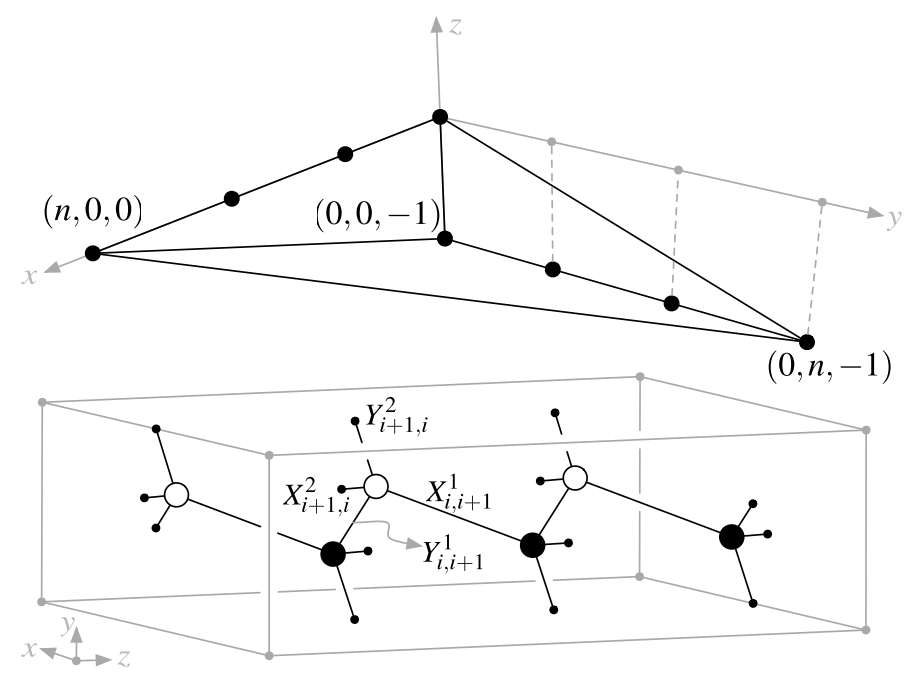

Figure 15: Toric diagram and crystal for $\left(\mathbb{C}^{2} / \mathbb{Z}_{n}\right)^{2}$.

We now turn to our crystal model. For simplicity, we restrict our attention to the $k=n$ cases. The toric diagram and the crystal for $\mathbb{C}^{2} / \mathbb{Z}_{n} \times \mathbb{C}^{2} / \mathbb{Z}_{n}$ are depicted in Figure 15. The gauge group of the abelian model is factorized as $\left(U(1)^{n} / U(1)_{D}\right)_{X} \times\left(U(1)^{n} / U(1)_{D}\right)_{Y}$. The matter fields $\left(X_{i, i+1}^{1}, X_{i+1, i}^{2}\right)$ are charged only under the first factor, $\left(U(1)^{n} / U(1)_{D}\right)_{X}$, as 


\begin{tabular}{l|cc} 
& $X_{i, i+1}^{1}$ & $X_{i+1, i}^{2}$ \\
\hline$Q_{i}$ & + & -
\end{tabular}$\quad(i=1, \cdots, n)$

with all other charges vanishing. The matter fields $\left(Y_{j, j+1}^{1}, Y_{j+1, j}^{2}\right)$ are charged only under the second factor, $\left(U(1)^{n} / U(1)_{D}\right)_{Y}$, in the same way as the $X$ fields. The super-potential of the crystal model is

$$
\mathcal{W}=\sum_{i=1}^{n}\left(X_{i, i+1}^{1} X_{i+1, i}^{2} Y_{i, i+1}^{1} Y_{i+1, i}^{2}-X_{i-1, i}^{1} X_{i, i-1}^{2} Y_{i, i+1}^{1} Y_{i+1, i}^{2}\right)
$$

from which we find the F-term conditions,

$$
\begin{gathered}
X_{i, i+1}^{1} X_{i+1, i}^{2}-X_{i-1, i}^{1} X_{i, i-1}^{2}=0 \\
Y_{j, j+1}^{1} Y_{j+1, j}^{2}-Y_{j-1, j}^{1} Y_{j, j-1}^{2}=0 .
\end{gathered}
$$

Comparing the field contents, gauge groups and F-term conditions with those of the D2-brane gauge theories, we note that $\mathcal{M}_{V}$ of the crystal model can be regarded as the product of the Higgs branch, $\mathcal{M}_{H}$, of the first description and the Higgs branch, $\widetilde{\mathcal{M}}_{H}$, of the dual description. ${ }^{1}$ In other words,

$$
\mathcal{M}_{V}(\text { crystal })=\mathcal{M}_{H} \times \widetilde{\mathcal{M}}_{H}
$$

Unlike in the D2-brane gauge theory, where the distinction between the Coulomb and Higgs branches is inevitable, the crystal model treats the two factors on an equal footing.

Note that, although the moduli space of vacua factorizes, the super-potential (4.13) has products of $X$ fields and $Y$ fields. It would be interesting to understand how the super-potential (4.13) arises from the dynamics of the D2-brane gauge theory.

\footnotetext{
${ }^{1}$ Similar Higgs branches also appear in the hyper-Kähler quotient description of the $d=3, \mathcal{N}=3$ CFT discussed in $[30,31,32]$.
} 


\section{Toric Duality}

It was observed in [11] that $C\left(T^{1,1}\right) \times \mathbb{C}$ admits two different crystals (See Figure 16 below). The result was based on the fast forward algorithm. Let us compare the abelian gauge theories of the two crystals.

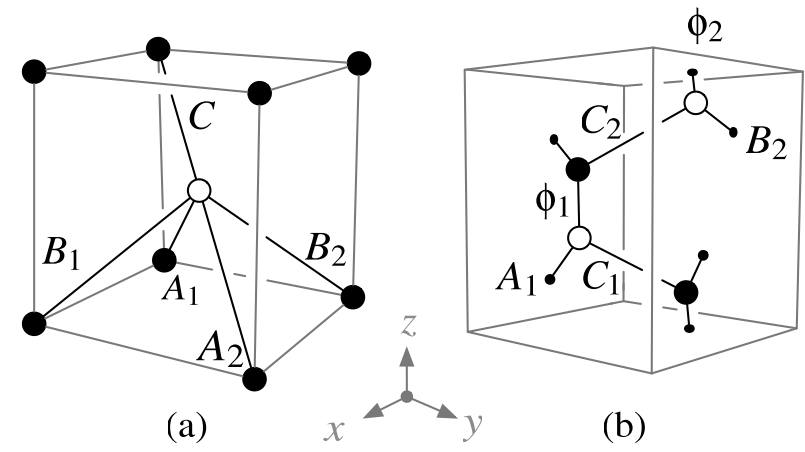

Figure 16: Two crystals for $C\left(T^{1,1}\right) \times \mathbb{C}$, reproduced from [11].

The field theory corresponding to crystal (a) has one independent gauge group. The charge assignment for the matter fields is as follows:

$$
\begin{array}{c|ccccc} 
& A_{1} & A_{2} & B_{1} & B_{2} & C \\
\hline Q & + & + & - & - & 0
\end{array} .
$$

The super-potential vanishes as usual since it has only two atoms. The gauge invariant variables parameterizing the moduli space of vacua are

$$
z_{1}=A_{1} B_{1}, \quad z_{2}=A_{1} B_{2}, \quad z_{3}=A_{2} B_{1}, \quad z_{4}=A_{2} B_{2}, \quad w=C
$$

which satisfy the relation $z_{1} z_{3}=z_{2} z_{4}$. The moduli space is clearly $C\left(T^{1,1}\right) \times \mathbb{C}$.

For crystal (b), all matter fields are neutral and the super-potential takes the form

$$
W=\phi_{1} A_{1} C_{1}-\phi_{2} A_{1} C_{1}+\phi_{2} B_{2} C_{2}-\phi_{1} B_{2} C_{2}
$$


from which we derive the F-term conditions:

$$
A_{1} C_{1}=B_{2} C_{2}, \quad \phi_{1}=\phi_{2}
$$

The moduli space is again $C\left(T^{1,1}\right) \times \mathbb{C}$.

Thus we see that the two crystals give two field theories describing the same toric singularity. The same kind of degeneracy of four-dimensional abelian gauge theories was discovered in [20], and was named toric duality. It was shown later that toric duality is realized in the non-abelian quiver gauge theories as Seiberg duality.

To investigate the fascinating possibility of "non-abelian" toric duality in M-theory, we will have to construct the "non-abelian" theory first, which is beyond the scope of this paper. In the rest of this section, we will show that, at least in the abelian context, toric duality of the crystal model is similar to that of the tiling model in many respects.

\subsection{GLSM charge matrix}

In the discussion of toric duality in the tiling model [20], the gauged linear sigma model (GLSM) description of the toric singularity plays a crucial role. The matter fields of the GLSM are precisely the perfect matchings $p_{\alpha}$. The charge matrix $Q$ of the GLSM can be divided into two parts, $Q=\left(Q_{F}, Q_{D}\right)^{T}$, where $Q_{F}$ is related to the F-term conditions of the (abelian) field theory while $Q_{D}$ originates form the D-term conditions. Let us briefly review how to construct $Q_{F}$ and $Q_{D}$ from the field theory. See [20] for details.

As we discussed in section 2, the perfect matchings solve the F-term conditions automatically via

$$
X_{i}=\prod_{\alpha} p_{\alpha}^{\left\langle X_{i}, p_{\alpha}\right\rangle}
$$

where $\left\langle X_{i}, p_{\alpha}\right\rangle$ equals 1 if $p_{\alpha}$ contains the bond $X_{i}$ and 0 otherwise. In general, the number of perfect matchings $\left\{p_{\alpha}\right\}$ can be larger than the number of fields $\left\{X_{i}\right\}$. Following [20], as an in- 
termediate step, we can choose a minimal set of independent fields $\left\{\tilde{X}_{m}\right\}$ after solving the F-term conditions. Define a matrix $T$ as

$$
T_{m \alpha}=\left\langle\tilde{X}_{m}, p_{\alpha}\right\rangle
$$

Then, $Q_{F}$ is determined as the cokernel of the $T$,

$$
\sum_{\alpha} T_{m \alpha} Q_{F \hat{m} \alpha}=0
$$

By construction, all fields $X_{i}$ are neutral under $Q_{F}$. They are only charged under the abelian gauge groups of the field theory, which are related to $Q_{D}$. Concretely, $Q_{D}$ assigns charges $\left(Q_{D}\right)_{a \alpha}$ to the perfect matchings $p_{\alpha}$ such that the charges of the abelian gauge theory $Q_{a i}$ is reproduced through (5.5).

As a consistency check, let us count the dimension of $\mathcal{M}_{V}$ of the GLSM. As discussed in section 4, the number of independent matter fields $\left\{\tilde{X}_{m}\right\}$ is $e-v+2$ and the number of relevant $U(1)$ gauge groups is $q=e-v-2$. Suppose that there are $p$ perfect matchings. The charge matrix $Q$ is then $\mathrm{a}(p-(e-v+2)+(e-v-2)) \times p=(p-4) \times p$ matrix, which implies that the dimension of the moduli space of vacua of the GLSM is four as expected.

The GLSM can be used to study blow-up of the toric singularity. The blow-ups are controlled by the FI parameters associated to the gauge groups. In the tiling/crystal models, since the GLSM charge matrix is constructed from the field theory underlying the tiling/crystal, not all FI parameters are allowed in the GLSM. The $Q_{D}$ part of the GLSM charge matrix can inherit the FI parameters from the gauge theory, but the $Q_{E}$ part is not allowed to have the FI parameters.

\section{2 $C\left(T^{1,1}\right) \times \mathbb{C}$ revisited}

Let us construct the GLSM charge matrices of the two crystals for $C\left(T^{1,1}\right) \times \mathbb{C}$. For crystal (a), all matter fields are independent since there is no super-potential. In terms of perfect matchings 
(Figure 17(a)), they are written as

$$
A_{1}=p_{1}, B_{2}=p_{3}, B_{1}=p_{4}, A_{2}=p_{6}, C=p_{0},
$$

which implies that the matrix $T$ is an identity matrix and that $Q_{F}=0$. Therefore, the GLSM charge matrix consists of only $Q_{D}$, which is identical to the charge of the abelian gauge theory:

$$
Q=\left(\begin{array}{llllll}
1 & -1 & -1 & 1 & 0 & ; \zeta
\end{array}\right)
$$

where we included the FI parameter $\zeta$ of the field theory.
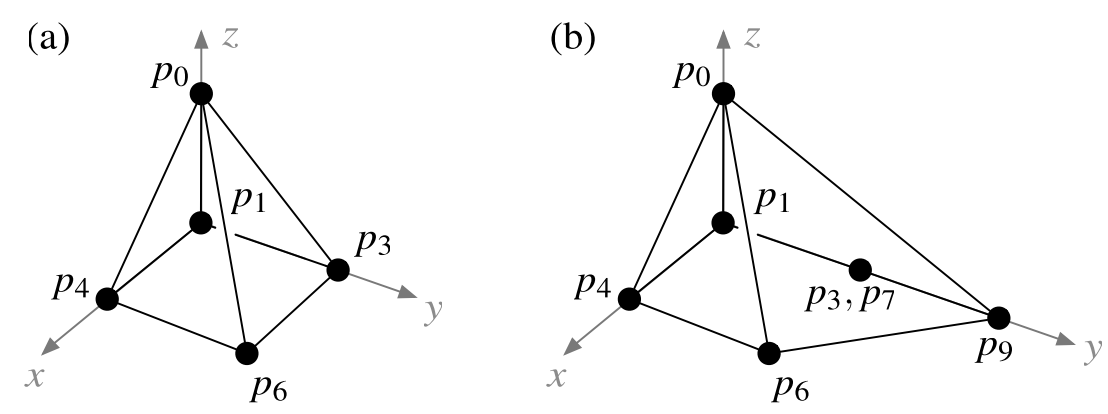

Figure 17: Perfect matchings of $C\left(T^{1,1}\right) \times \mathbb{C}$ and $\mathrm{SPP} \times \mathbb{C}$.

For crystal (b), the independent matter fields are chosen as $\left\{\phi_{1}, C_{1}, A_{1}, B_{2}\right\}$ after solving the F-term conditions (5.4). These matter fields are written in terms of the perfect matching as

$$
\phi_{1}=p_{0}, C_{1}=p_{1} p_{3}, A_{1}=p_{4} p_{6}, B_{2}=p_{3} p_{6}
$$


which leads to

$$
T=\left(\begin{array}{lllll}
0 & 0 & 0 & 0 & 1 \\
1 & 1 & 0 & 0 & 0 \\
0 & 0 & 1 & 1 & 0 \\
0 & 1 & 0 & 1 & 0
\end{array}\right) \leftarrow\left(\begin{array}{c|ccccc} 
& p_{1} & p_{3} & p_{4} & p_{6} & p_{0} \\
\hline \phi_{1} & 0 & 0 & 0 & 0 & 1 \\
C_{1} & 1 & 1 & 0 & 0 & 0 \\
A_{1} & 0 & 0 & 1 & 1 & 0 \\
B_{2} & 0 & 1 & 0 & 1 & 0
\end{array}\right)
$$

The charge matrix $Q_{F}$ (cokernel of $T$ ) is

$$
Q_{F}=\left(\begin{array}{lllll}
1 & -1 & -1 & 1 & 0
\end{array}\right)
$$

The charge matrix $Q_{D}$ is absent since all the matter fields in the theory are neutral. So, the GLSM charge matrix becomes

$$
Q=\left(\begin{array}{lllll}
1 & -1 & -1 & 1 & 0 ; 0
\end{array}\right)
$$

where the last entry means that the FI term is absent.

We therefore conclude that the GLSM charge matrices of the two theories are identical, but their physical origin is different. One comes from the D-term conditions, and the other from the Fterm conditions. This phenomenon, called F-D ambiguity in [20], lies at the heart of toric duality. A crucual difference is that an FI term can be introduced in crystal (a), but not in crystal (b). In the next section, we will see what difference they make when we study possible blow-ups of the singularity.

\subsection{Another example}

We consider another illustrative example of toric duality, namely, $S P P \times \mathbb{C}$. We have again two different crystals depicted in Figure 18. For crystal (a), the field theory has seven matter fields 

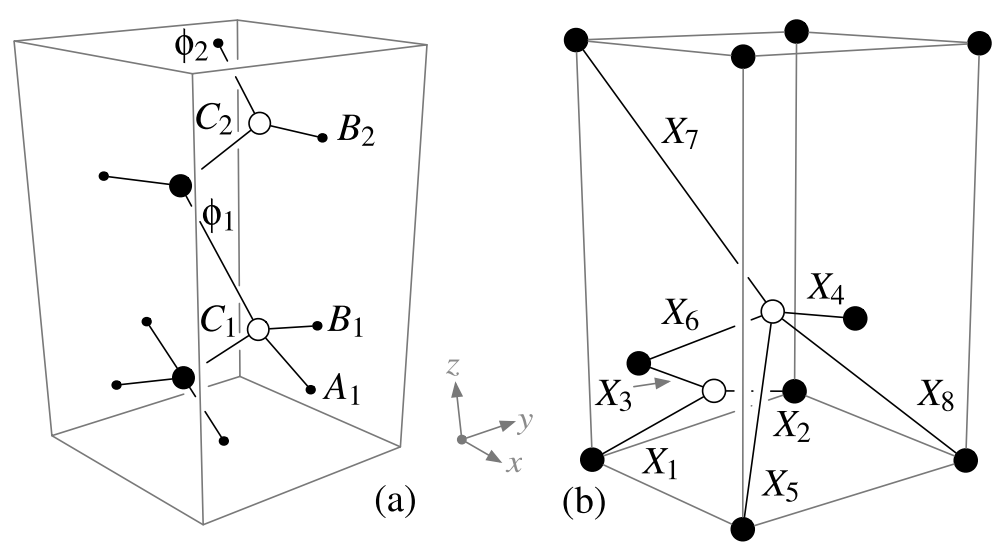

Figure 18: Two crystals for $\mathrm{SPP} \times \mathbb{C}$.

whose charges under the relevant $U(1)$ gauge group are assigned as

$$
\begin{array}{c|ccccccc} 
& \phi_{1} & \phi_{2} & A_{1} & B_{1} & B_{2} & C_{1} & C_{2} \\
\hline Q & 0 & 0 & 0 & + & - & - & +
\end{array} .
$$

The super-potential $W$ takes the form

$$
W=\phi_{1} A_{1} B_{1} C_{1}-\phi_{2} A_{1} B_{1} C_{1}+\phi_{2} B_{2} C_{2}-\phi_{2} B_{2} C_{2}
$$

which gives the F-term conditions

$$
A_{1} B_{1} C_{1}=B_{2} C_{2}, \phi_{1}=\phi_{2}
$$

After solving these F-term conditions, we choose the independent matter fields to be $\left\{\phi_{1}, C_{1}, A_{1}, B_{1}, C_{2}\right\}$. They are written in terms of perfect matchings (Figure 17(b)) as

$$
\phi_{1}=p_{0}, C_{1}=p_{1} p_{3}, A_{1}=p_{4} p_{6}, B_{1}=p_{7} p_{9}, C_{2}=p_{1} p_{4} p_{7}
$$


from which we find

$$
T=\left(\begin{array}{ccccccc}
0 & 0 & 0 & 0 & 0 & 0 & 1 \\
1 & 1 & 0 & 0 & 0 & 0 & 0 \\
0 & 0 & 1 & 1 & 0 & 0 & 0 \\
0 & 0 & 0 & 0 & 1 & 1 & 0 \\
1 & 0 & 1 & 0 & 1 & 0 & 0
\end{array}\right) \text { and } Q_{F}=\left(\begin{array}{ccccccc}
p_{1} & p_{3} & p_{4} & p_{6} & p_{7} & p_{9} & p_{0} \\
1 & -1 & 0 & 0 & -1 & 1 & 0 \\
1 & -1 & -1 & 1 & 0 & 0 & 0
\end{array}\right)
$$

The gauge charges of matter fields are reproduced if we choose $Q_{D}$ to be

$$
Q_{D}=\left(\begin{array}{lllllll}
0 & -1 & 0 & 0 & 1 & 0 & 0
\end{array}\right)
$$

Altogether, the GLSM charge matrix $Q$ for the crystal (a) is given by

$$
Q=\left(\begin{array}{cccccccc}
1 & -1 & 0 & 0 & -1 & 1 & 0 & ; 0 \\
1 & -1 & -1 & 1 & 0 & 0 & 0 & ; 0 \\
0 & -1 & 0 & 0 & 1 & 0 & 0 & ; \zeta
\end{array}\right)
$$

For crystal (b), the charges of eight matter fields for the abelian theory are

$$
\begin{array}{c|cccccccc} 
& X_{1} & X_{2} & X_{3} & X_{4} & X_{5} & X_{6} & X_{7} & X_{8} \\
\hline Q_{1} & 0 & 0 & 0 & - & - & + & 0 & + \\
Q_{2} & - & + & 0 & - & 0 & + & 0 & 0
\end{array} .
$$

We can also read off the super-potential from the four atoms in the crystal,

$$
W=X_{1} X_{2} X_{3}+X_{4} X_{5} X_{6} X_{7} X_{8}-X_{3} X_{4} X_{6}-X_{1} X_{2} X_{5} X_{7} X_{8}
$$

which gives the F-term conditions

$$
X_{5} X_{7} X_{8}=X_{3}, X_{1} X_{2}=X_{4} X_{6}
$$


With gauge invariant monomials

$$
\begin{aligned}
& z_{1}=X_{5} X_{8}, \quad z_{2}=X_{2} X_{4} X_{8}, \quad z_{3}=X_{1} X_{5} X_{6}, \\
& z_{4}=X_{3}, \quad z_{5}=X_{7}, \quad w=X_{1} X_{2}\left(=X_{4} X_{6}\right),
\end{aligned}
$$

the moduli space of vacua can be described as

$$
z_{1} w^{2}=z_{2} z_{3}, \quad z_{1} z_{5}=z_{4}
$$

which is an algebraic description of $S P P \times \mathbb{C}$ singularity. In terms of perfect matchings, the matter fields are written by

$$
\begin{gathered}
X_{1}=p_{1} p_{3}, X_{2}=p_{7} p_{9}, X_{3}=p_{4} p_{6} p_{0}, \\
X_{4}=p_{3} p_{9}, X_{5}=p_{4}, X_{6}=p_{1} p_{7}, X_{7}=p_{0}, X_{8}=p_{6} .
\end{gathered}
$$

By the method introduced in the previous subsection, we can construct the GLSM charge matrix Q

$$
Q=\left(\begin{array}{cccccccc}
p_{1} & p_{3} & p_{4} & p_{6} & p_{7} & p_{9} & p_{0} & \\
1 & -1 & 0 & 0 & -1 & 1 & 0 & ; 0 \\
1 & -1 & -1 & 1 & 0 & 0 & 0 & ; \zeta_{1} \\
0 & -1 & 0 & 0 & 1 & 0 & 0 & ; \zeta_{2}
\end{array}\right)
$$

which is the same as (5.20) except that the second row in this case comes from a D-term rather than an F-term. These two crystals are therefore toric dual. 


\section{Partial resolution}

As we have seen in previous section, the crystal model encodes the geometry of the CY singularity via Kähler quotient (GLSM). We can take Kähler deformations of the singularity by introducing the blow-up parameters $\zeta_{a}$ to the moment maps. The moment maps with generic parameters would resolve the singularity completely. For special values of the parameters, some residual singularities may survive. This procedure is known as partial resolutions.

Partial resolutions in the context of $\mathrm{AdS}_{5} / \mathrm{CFT}_{4}$ has been extensively discussed, for example, in [19]. Let us recall how D-branes "know" about the smoothing. When a D3-brane probes the CY singularity, the closed string modes in the twisted sector couple to open string modes as the FI terms on the world-volume gauge theory. Turning on the FI terms induces vevs for some of the charged matter fields, which leads to (partial) gauge symmetry breaking via Higgs mechanism. Keeping light fields only, one obtains the low energy effective theory which describe the D3-brane sitting at the residual singularity.

Partial resolution in the tiling model was studied most systematically in [33]. In the tiling diagram, partial resolutions results in taking off the edges corresponding to matter fields which acquire the vacuum expectation value. It results in another tiling diagram consistent with the remaining "daughter" singularity. The most efficient method of determining which edge to remove utilize the amoeba projection and the perfect matchings [33]. Here, we will use the older approach of [20] based on the GLSM of perfect matchings, since it shows the connection to the abelian gauge theory more clearly. Let us briefly summarize the procedure.

We begin by choosing a vertex of the toric diagram we would like to delete. The vertex should be at a corner of the toric diagram; otherwise, the remaining toric diagram will not be convex. Then we consider the moment map (D-term) equation of the GLSM with all allowed FI parameters included. The M-theory origin of the FI parameters is not clear, but we proceed with the assumption that they exist. We look for a solution to the moment map equation with a non-zero vev for the perfect matching sitting on the vertex to be deleted. The relation between matter fields $\left\{X_{i}\right\}$ of the gauge theory and the perfect matchings $p_{\alpha}$ of the GLSM then determines which bonds of the 
crystal should be removed by the partial resolution. Let us work out several illustrative examples in detail.

\section{1 $\mathbb{C}^{3} /\left(\mathbb{Z}_{2} \times \mathbb{Z}_{2}\right) \times \mathbb{C} \rightarrow \mathrm{SPP} \times \mathbb{C}$}

We first consider the field theory for the crystal $\mathbb{C}^{3} /\left(\mathbb{Z}_{2} \times \mathbb{Z}_{2}\right) \times \mathbb{C}$. See Figure 7 , where the matter fields with their charges and the super-potential are given. The perfect matchings $p_{\alpha}$ of this crystal are shown in Figure 19 below.

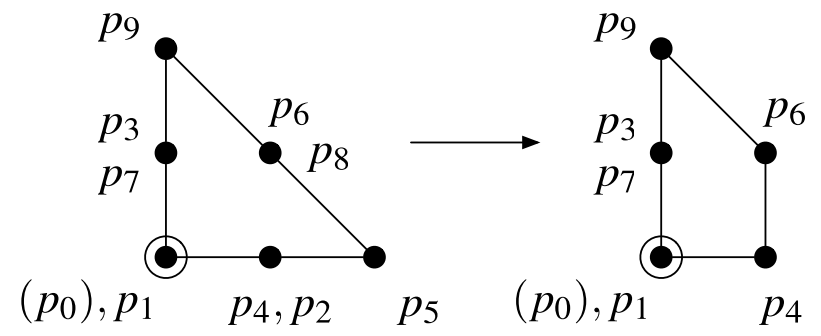

(a)

(b)

Figure 19: (a) Perfect matchings of $\mathbb{C}^{3} /\left(\mathbb{Z}_{2} \times \mathbb{Z}_{2}\right) \times \mathbb{C}$ projected onto the $(x, y)$-plane. All perfect matchings except $p_{0}$ lie on the plane. (b) Perfect matchings of $\mathrm{SPP} \times \mathbb{C}$ after partial resolution, which is identical to Figure 17(b).

In terms of the perfect matchings $p_{\alpha}$, the matter fields are written as

$$
\begin{aligned}
& A_{1}=p_{4} p_{5} p_{6}, B_{1}=p_{7} p_{8} p_{9}, C_{1}=p_{1} p_{2} p_{3}, \phi_{1}=p_{0} \\
& A_{2}=p_{2} p_{5} p_{8}, B_{2}=p_{3} p_{6} p_{9}, C_{2}=p_{1} p_{4} p_{7}, \phi_{2}=p_{0} .
\end{aligned}
$$


Following the procedure of subsection 5.1, we obtain the GLSM charge matrix,

$$
Q\left(\mathbb{C}^{3} /\left(\mathbb{Z}_{2} \times \mathbb{Z}_{2}\right) \times \mathbb{C}\right)=\left(\begin{array}{ccccccccccc}
p_{1} & p_{2} & p_{3} & p_{4} & p_{5} & p_{6} & p_{7} & p_{8} & p_{9} & p_{0} & \\
1 & 0 & -1 & 0 & 0 & 0 & -1 & 0 & 1 & 0 & ; 0 \\
1 & -1 & 0 & 0 & 0 & 0 & -1 & 1 & 0 & 0 & ; 0 \\
1 & 0 & -1 & -1 & 0 & 1 & 0 & 0 & 0 & 0 & ; 0 \\
1 & -1 & 0 & -1 & 1 & 0 & 0 & 0 & 0 & 0 & ; 0 \\
-1 & 1 & 1 & 0 & 0 & -1 & 0 & 0 & 0 & 0 & ; \zeta_{1} \\
0 & 0 & -1 & 0 & 0 & 0 & 1 & 0 & 0 & 0 & ; \zeta_{2}
\end{array}\right)
$$

Compared with the GLSM charge matrix of $d=4, \mathcal{N}=1$ gauge theory on a D3-brane probing $\mathbb{C}^{3} /\left(\mathbb{Z}_{2} \times \mathbb{Z}_{2}\right)$, the crucial difference is that our present model can have at most two FI parameters, whereas the D3-brane theory has three. In what follows, we will show that due to the lack of FI parameters, the M2-brane theory cannot capture all possible partial resolutions allowed in the D3-brane theory.

The toric diagram of $\mathbb{C}^{3} /\left(\mathbb{Z}_{2} \times \mathbb{Z}_{2}\right) \times \mathbb{C}$ in Figure 19 suggests that we can remove a point, say $p_{5}$, to obtain a partially resolved singularity $\mathrm{SPP} \times \mathbb{C}$. Let us check whether such a partial resolution can be realized in the GLSM and the abelian theory with a suitable choice of the FI terms.

In the GLSM, we should solve the corresponding moment map equation,

$$
Q \vec{y}=\left(0,0,0,0, \zeta_{1}, \zeta_{2}\right)^{\mathrm{T}},
$$

where $y_{\alpha}$ denotes $\left|p_{\alpha}\right|^{2}$. Since the fields of the GLSM are the perfect matchings, we know what to expect of the partial resolution. We want to remove $p_{5}$ from the toric diagram, so it should get a vev. On the other hand, the perfect matchings unaffected by the resolution, $\left\{p_{1}, p_{3}, p_{7}, p_{9}\right\}$, should not get vevs. The unique solution satisfying these requirements is

$$
\vec{y}=\left(0, \zeta_{1}, 0,0, \zeta_{1}, 0,0, \zeta_{1}, 0,0\right)^{\mathrm{T}},
$$


with $\zeta_{2}=0$. We find that, in addition to $p_{5}, p_{2}$ and $p_{8}$ also get vevs. To summarize, we obtain the toric diagram for $\mathrm{SPP} \times \mathbb{C}$ by removing $p_{2}, p_{5}, p_{8}$; see Figure $19(\mathrm{a}, \mathrm{b})$.

Let us translate these results into the field theory language. The D-term equations with the FI terms are ${ }^{2}$

$$
\begin{aligned}
& \left|C_{1}\right|^{2}-\left|C_{2}\right|^{2}-\left|A_{1}\right|^{2}+\left|A_{2}\right|^{2}=\zeta_{1} \\
& \left|B_{1}\right|^{2}-\left|B_{2}\right|^{2}-\left|C_{1}\right|^{2}+\left|C_{2}\right|^{2}=\zeta_{2} .
\end{aligned}
$$

When we turn on FI term $\zeta_{1}$ only, the point we choose on the moduli space of vacua is represented by the vev

$$
A_{2}=\zeta_{1}^{1 / 2}
$$

with the others vanishing, since the matter field $A_{2}$ is written as products only of resolved perfect matchings $\left\{p_{2}, p_{5}, p_{8}\right\}$. After integrating out massive modes, the low energy theory is governed by the super-potential

$$
W=\phi_{1}\left(A_{1} B_{1} C_{1}-B_{2} C_{2}\right)-\phi_{2}\left(A_{1} B_{1} C_{1}-B_{2} C_{2}\right),
$$

with one relevant $U(1)$ gauge symmetry under which the matter fields are charged as

$$
\begin{array}{c|ccccccc} 
& A_{1} & B_{1} & C_{1} & \phi_{1} & B_{2} & C_{2} & \phi_{2} \\
\hline Q & 0 & + & - & 0 & - & + & 0
\end{array} .
$$

This theory is nothing but the gauge theory for crystal (a) in Figure 18. Note that this crystal can be obtained simply by eliminating the bond $A_{2}$ in the crystal for $\mathbb{C}^{3} /\left(\mathbb{Z}_{2} \times \mathbb{Z}_{2}\right) \times \mathbb{C}$; compare Figures 13 and 18(a).

\footnotetext{
${ }^{2}$ We use the same notation for the FI parameters of the GLSM and those of the abelian gauge theory, but in general their numerical values are not the same.
} 


\section{2 $\quad \mathrm{SPP} \times \mathbb{C} \rightarrow C\left(T^{1,1}\right) \times \mathbb{C}$ or $\mathbb{C}^{2} / \mathbb{Z}_{2} \times \mathbb{C}^{2}$}

We now ask whether we can further resolve $\mathrm{SPP} \times \mathbb{C}$ to reach the daughter singularities $C\left(T^{1,1}\right) \times \mathbb{C}$ or $\mathbb{C}^{2} / \mathbb{Z}_{2} \times \mathbb{C}^{2}$. Recall that in subsection 5.3 , we analyzed the field theories for the two crystals for $\mathrm{SPP} \times \mathbb{C}$ that are toric dual to each other. We analyze both of them in turn.

For crystal (a) in Figure 18, let us summarize the field theory information here for convenience. We can express the matter fields in terms of perfect matchings as

$$
\begin{array}{r}
A_{1}=p_{4} p_{6}, B_{1}=p_{7} p_{9}, C_{1}=p_{1} p_{3}, \phi_{1}=p_{0} \\
B_{2}=p_{3} p_{6} p_{9}, C_{2}=p_{1} p_{4} p_{7}, \phi_{2}=p_{0},
\end{array}
$$

and the GLSM charge matrix is given by

$$
Q(\mathrm{SPP} \times \mathbb{C})=\left(\begin{array}{cccccccc}
p_{1} & p_{3} & p_{4} & p_{6} & p_{7} & p_{9} & p_{0} & \\
1 & -1 & 0 & 0 & -1 & 1 & 0 & ; 0 \\
1 & -1 & -1 & 1 & 0 & 0 & 0 & ; 0 \\
0 & -1 & 0 & 0 & 1 & 0 & 0 & ; \zeta
\end{array}\right)
$$

In the GLSM, we begin again by solving the moment map equation,

$$
Q \vec{y}=(0,0, \zeta)^{\mathrm{T}}
$$

To obtain $\mathcal{C}\left(T^{1,1}\right) \times \mathbb{C}$, we require that $p_{9}$ get a vev. It is easy to find a solution,

$$
\vec{y}=(0,0,0,0, \zeta, \zeta, 0)^{\mathrm{T}}
$$

implying that $p_{7}$ also gets a vev. This leads to a toric diagram for $\mathcal{C}\left(T^{1,1}\right) \times \mathbb{C}$ with the surviving perfect matchings $\left\{p_{0}, p_{1}, p_{3}, p_{4}, p_{6}\right\}$. On the gauge theory side, the $\mathrm{D}$-term equation is 


$$
\left|B_{1}\right|^{2}-\left|B_{2}\right|^{2}-\left|C_{1}\right|^{2}+\left|C_{2}\right|^{2}=\zeta
$$

From (6.9), we see that $B_{1}=p_{7} p_{9}$ is the only field to acquire a vev,

$$
B_{1}=\zeta^{1 / 2}
$$

The low energy theory becomes the gauge theory for crystal (b) in Figure 16. As a check, note that this can be obtained from crystal (a) in Figure 18 by taking off the bond $B_{1} .^{3}$

Next, we try to obtain $\mathbb{C}^{2} / \mathbb{Z}_{2} \times \mathbb{Z}_{2}$ by giving a vev to $p_{6}$. However, a solution to (6.11) with a non-zero vev for $p_{6}$ does not exist. All other solutions of (6.11) correspond to the complete resolution of the singularity or the partial resolution to $C\left(T^{1,1}\right) \times \mathbb{C}$. Thus we see that unlike the D3-brane theory, the M2-brane theory probing $\mathbb{C}^{3} /\left(\mathbb{Z}_{2} \times \mathbb{Z}_{2}\right) \times \mathbb{C}$, does not have room for enough FI parameters to allow partial resolution to all conceivable daughter singularities.

We now turn to study partial resolutions of crystal (b) in Figure 18. We again summarize the field theory information here for convenience. The GLSM charge matrix is

$$
\tilde{Q}(\mathrm{SPP} \times \mathbb{C})=\left(\begin{array}{cccccccc}
p_{1} & p_{3} & p_{4} & p_{6} & p_{7} & p_{9} & p_{0} & \\
1 & -1 & 0 & 0 & -1 & 1 & 0 & ; 0 \\
1 & -1 & -1 & 1 & 0 & 0 & 0 & ; \zeta_{1} \\
0 & -1 & 0 & 0 & 1 & 0 & 0 & ; \zeta_{2}
\end{array}\right)
$$

Compared to (6.10), we have an additional FI parameter. We will see that it opens up new possibilities for partial resolutions. The D-term equations become

$$
\begin{aligned}
-\left|X_{3}\right|^{2}-\left|X_{4}\right|^{2}-\left|X_{5}\right|^{2}+\left|X_{6}\right|^{2}+\left|X_{8}\right|^{2} & =\zeta_{1} \\
-\left|X_{1}\right|^{2}+\left|X_{2}\right|^{2}-\left|X_{4}\right|^{2}+\left|X_{6}\right|^{2} & =\zeta_{2} .
\end{aligned}
$$

\footnotetext{
${ }^{3}$ To avoid confusion, note that the coordinate axes are oriented differently in the two figures.
} 
The super-potential for this theory takes the form

$$
W=X_{1} X_{2} X_{3}+X_{4} X_{5} X_{6} X_{7} X_{8}-X_{3} X_{4} X_{6}-X_{1} X_{2} X_{5} X_{7} X_{8}
$$

In terms of perfect matchings, the matter fields can be written as

$$
\begin{gathered}
X_{1}=p_{1} p_{3}, X_{2}=p_{7} p_{9}, X_{3}=p_{4} p_{6} p_{0}, \\
X_{4}=p_{3} p_{9}, X_{5}=p_{4}, X_{6}=p_{1} p_{7}, X_{7}=p_{0}, X_{8}=p_{6} .
\end{gathered}
$$

Solving the moment map equations with the GLSM charge matrix (6.15), we find two solutions. The first one is

$$
\vec{y}=\left(0,-\zeta_{1}, 0,0,0,-\zeta_{2}, 0\right)^{\mathrm{T}},
$$

with $\zeta_{1}=\zeta_{2}(\neq 0)$. Since $p_{3}, p_{9}$ are resolved, only the matter field $X_{4}$ acquires a vev

$$
X_{4}=\left(-\zeta_{1}\right)^{1 / 2}
$$

Replacing $X_{4}$ by its vev in (6.17), we find that $X_{3}$ and $X_{6}$ get F-term masses. In fact, integrating out the massive modes make the super-potential vanish completely. The remaining theory with one gauge group and five fields $\left\{X_{1}, X_{2}, X_{5}, X_{7}, X_{8}\right\}$ is identical to that of the crystal $C\left(T^{1,1}\right) \times \mathbb{C}$ in Figure 16(a), once we relabel the fields by

$$
X_{1} \rightarrow A_{1}, \quad X_{2} \rightarrow B_{2}, \quad X_{5} \rightarrow B_{1}, \quad X_{7} \rightarrow C, \quad X_{8} \rightarrow A_{2}
$$

The charges $Q$ in (5.1) is related to $Q_{1}$ and $Q_{2}$ in (5.21) by $Q=Q_{1}-Q_{2}$.

Pictorially, elimination of the bond corresponding to $X_{4}$ produces a bi-valent atom which can be regarded as a mass term. Integrating out the massive modes translates into shrinking the bi-valent atom and the bonds attached to it. The remaining crystal coincides with Figure 16(a). 
The other solution of the moment map equation is given by

$$
\vec{y}=\left(0,0,0, \zeta_{1}, 0,0,0\right)^{\mathrm{T}}, \quad \zeta_{2}=0
$$

In the toric diagram, removing $p_{6}$ leads to the daughter singularity $\mathbb{C}^{2} / \mathbb{Z}_{2} \times \mathbb{C}^{2}$. We can check it at the level of the field theory. From (6.18), we see that $X_{8}$ is the only field to acquire a vev through $p_{6}$.

$$
X_{8}=\zeta_{1}^{1 / 2}
$$

The low energy theory around this vacuum is now governed by the super-potential read off from $(6.17)$

$$
W=X_{1} X_{2} X_{3}+X_{4} X_{5} X_{6} X_{7}-X_{3} X_{4} X_{6}-X_{1} X_{2} X_{5} X_{7}
$$

The charges under the remaining gauge group are given by

$$
\begin{array}{c|ccccccc} 
& X_{1} & X_{2} & X_{3} & X_{4} & X_{5} & X_{6} & X_{7} \\
\hline Q & - & + & 0 & - & 0 & + & 0
\end{array} .
$$

This agrees precisely with the field theory for the crystal $\mathbb{C}^{2} / \mathbb{Z}_{2} \times \mathbb{C}^{2}$ summarized in Figure 11 , if we relabel the fields by

$$
X_{3} \rightarrow \phi, X_{1} \rightarrow Y_{2}, X_{2} \rightarrow Y_{1}, X_{4} \rightarrow Y_{4}, X_{6} \rightarrow Y_{3}, X_{5} \rightarrow X_{1}, X_{7} \rightarrow X_{2}
$$

One can also verify that removing the bond $X_{8}$ from the crystal (b) in Figure 18 and changing the basis for the unit cell of the crystal give the crystal in Figure 11.

Figure 20 summarizes all the results we have obtained so far. The solid arrows denote partial resolutions and the dashed bi-directional arrows denote toric duality. 


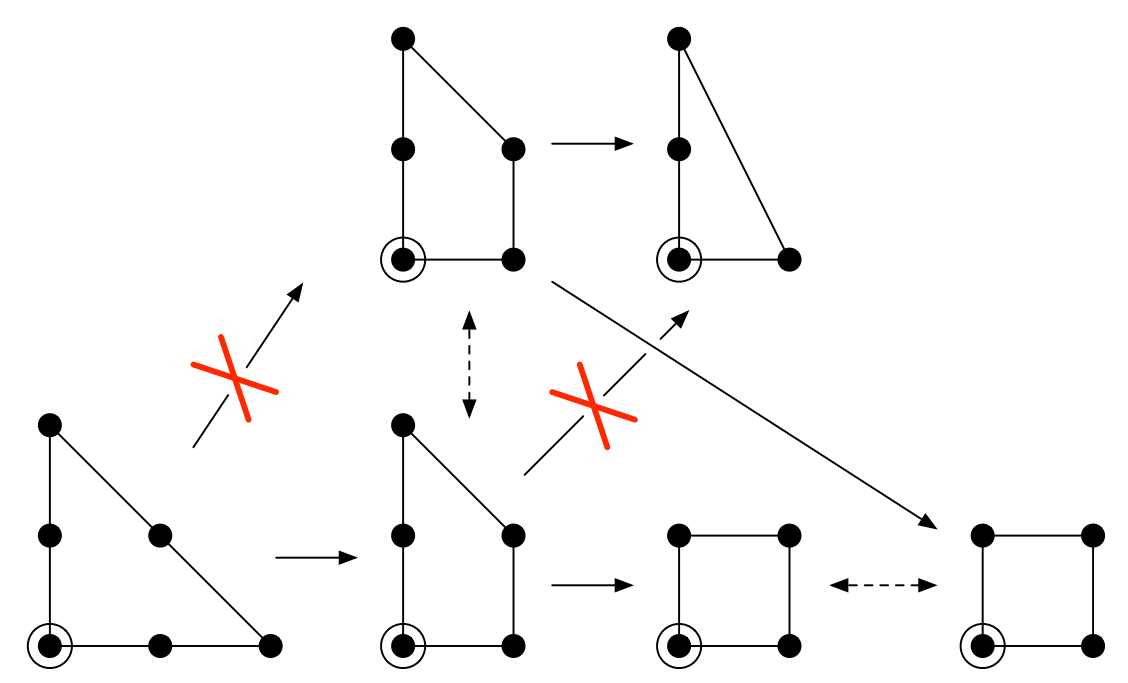

Figure 20: Partial resolutions and toric dualities.

\section{New RG Flows?}

In $\mathrm{AdS}_{5} / \mathrm{CFT}_{4}$, there are two famous holographic RG flows. One is the Klebanov-Witten (KW) flow [21] from the orbifold $\mathbb{C}^{2} / \mathbb{Z}_{2} \times \mathbb{C}^{2}$ to the conifold $C\left(T^{1,1}\right)$. The other one is the PilchWarner (PW) flow [34, 35], $\mathcal{N}=4 \rightarrow \mathcal{N}=1^{*}$, and orbifolds thereof. To our knowledge, nearly all examples of $\mathrm{RG}$ flows in $\mathrm{AdS}_{4} / \mathrm{CFT}_{3}$ discussed in the literature $[36,37,38,39,40,41,42]$ are analogous to the PW flow rather than the KW flow. ${ }^{4}$ We will now use our crystal model to argue that new RG flows of the KW type may exist.

\subsection{Klebanov-Witten flow revisited}

We begin with a short review of the KW flow with emphasis on its tiling model interpretation. The super-potential of the $\mathbb{C}^{2} / \mathbb{Z}_{2} \times \mathbb{C}$ orbifold is encoded in the tiling (a) in Figure 21 .

$$
W_{\mathrm{UV}}=\operatorname{Tr}\left(\phi_{1} A_{1} B_{1}-\phi_{2} B_{1} A_{1}+\phi_{2} B_{2} A_{2}-\phi_{1} A_{2} B_{2}\right) .
$$

\footnotetext{
${ }^{4}$ An exception is Ref. [2], where it was argued that an RG flow of the KW type from $\left(\mathbb{C}^{2} / \mathbb{Z}_{2}\right)^{2}$ to $C\left(Q^{1,1,1}\right)$ exist. It does not seem to be allowed in our crystal model.
} 
The RG-flow (massive deformation) is triggered by the so-called twisted mass term,

$$
\Delta W_{\mathrm{UV}}=\frac{m}{2} \operatorname{Tr}\left(\phi_{1}^{2}-\phi_{2}^{2}\right)
$$

Integrating out $\phi_{1}$ and $\phi_{2}$ leaves the super-potential

$$
W_{\mathrm{IR}}=\frac{1}{m} \operatorname{Tr}\left(A_{1} B_{1} A_{2} B_{2}-B_{1} A_{1} B_{2} A_{2}\right) .
$$

Can we understand not only the end-points of the flow but the entire flow in the tiling model? The mass term (7.2) causes a problem, because in the tiling model a matter field can appear in a superpotential term at most once. We can circumvent this difficulty by considering a slightly different UV theory which flows to the same theory in the IR. The new UV theory, described by the tiling (b) in Figure 21, has the super-potential,

$$
\widetilde{W}_{\mathrm{UV}}=\operatorname{Tr}\left(\phi_{1}^{+} A_{1} B_{1}-\phi_{2}^{-} B_{1} A_{1}+\phi_{2}^{+} B_{2} A_{2}-\phi_{1}^{-} A_{2} B_{2}\right)-\operatorname{Tr}\left(\phi_{1}^{+} \phi_{1}^{-}-\phi_{2}^{+} \phi_{2}^{-}\right)
$$

Integrating out $\phi_{1}^{ \pm}$and $\phi_{2}^{ \pm}$leads to the same super-potential as (7.3) in the IR.

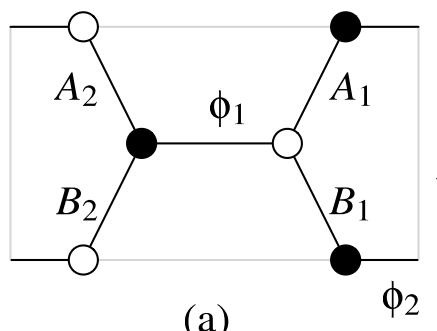

(a)

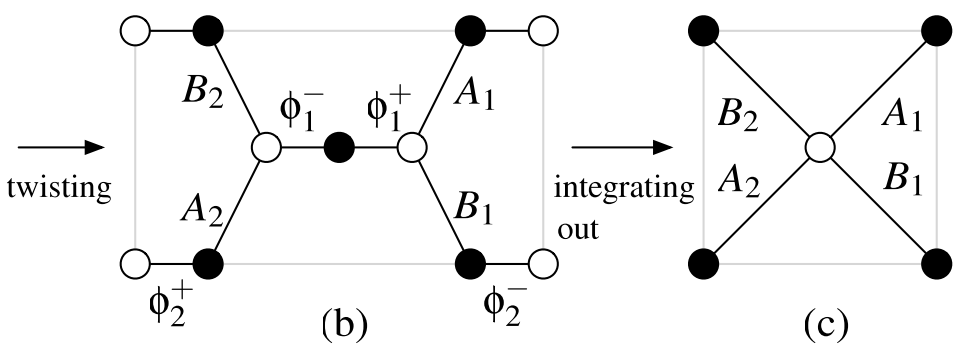

(b) (c)

Figure 21: Klebanov-Witten flow in the tiling model.

Although the new UV theory is not quite the same as the original mass-deformed theory, it is useful for a few reasons. First, not only the theory itself, but also the process of integrating out massive fields can be described in the tiling model. It corresponds to removing the mass terms (bivalent atoms) by shrinking the bonds attached to it; see Figure 21(b, c). Second, it can be derived from the original, undeformed, theory in a simple and systematic way. 
Recall that the inverse algorithm of the tiling model produces the tiling graph from the set of intersecting 1-cycles (zig-zag paths). The transition from tiling (a) to (b) in Figure 21 can be interpreted as an extra twisting of the 1-cycles. See Figure 22 below. Note that the twisting produces a new vertex corresponding to the mass term, flips the colors of the vertices on the left half, and exchanges the locations of $A_{2}$ and $B_{2}$.

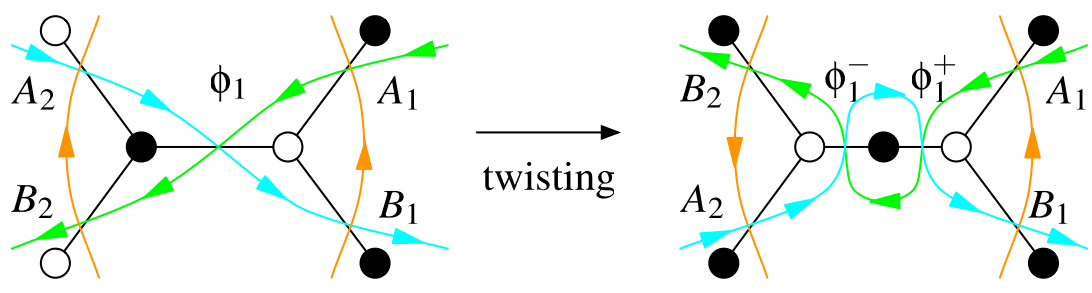

Figure 22: Twisting.

To conclude, given the tiling graph for the UV theory, the twisting interpretation offers an intuitive and efficient way to find out the tiling graph for the IR theory.

\subsection{M-theory flows}

We now study massive deformation of the abelian gauge theory in the crystal model. We find two examples which resemble the KW flow. The twisting picture we discussed in the previous subsection again gives an intuitive picture of the flow. Whether these flows really exist in the "non-abelian" theory is a very interesting and important problem. Although a direct analysis of the "non-abelian" theory is not feasible at present, its AdS/CFT dual description on the supergravity side should be possible in a way similar to the analysis of the KW flow [43]. A detailed study of the supergravity flows will be reported elsewhere [44].

Our first example is the flow from the orbifold $\mathbb{C}^{3} /\left(\mathbb{Z}_{2} \times \mathbb{Z}_{2}\right) \times \mathbb{C}$ to $D_{3}$. We recall from section 3 that the $\mathbb{C}^{3} /\left(\mathbb{Z}_{2} \times \mathbb{Z}_{2}\right) \times \mathbb{C}$ model has the charge assignment,

\begin{tabular}{c|cccccccc} 
& $\phi_{1}$ & $\phi_{2}$ & $A_{1}$ & $A_{2}$ & $B_{1}$ & $B_{2}$ & $C_{1}$ & $C_{2}$ \\
\hline$Q_{1}$ & 0 & 0 & + & - & - & + & 0 & 0 \\
$Q_{2}$ & 0 & 0 & - & + & 0 & 0 & + & -
\end{tabular}


and the super-potential

$$
W=\phi_{1}\left(A_{1} B_{1} C_{1}-A_{2} B_{2} C_{2}\right)-\phi_{2}\left(A_{1} B_{1} C_{1}-A_{2} B_{2} C_{2}\right) .
$$

If we add the twisted mass term,

$$
\Delta W=\frac{m}{2}\left(\phi_{1}^{2}-\phi_{2}^{2}\right)
$$

and integrate out $\phi_{1}$ and $\phi_{2}$, then the resulting theory has a vanishing super-potential, while the charges of the remaining fields remain unchanged. This is nothing but the abelian theory of $D_{3}$.

Let us check how the moduli space of vacua changes. Recall that the $\mathbb{C}^{3} /\left(\mathbb{Z}_{2} \times \mathbb{Z}_{2}\right) \times \mathbb{C}$ orbifold has the algebraic description $z_{1} z_{2} z_{3}=w^{2}$ with an unconstrained variable $\phi$; see the paragraph containing (4.4). After the RG flow, the variable $\phi$ disappear and the algebraic equation is deformed to $z_{1} z_{2} z_{3}=w_{1} w_{2}$.

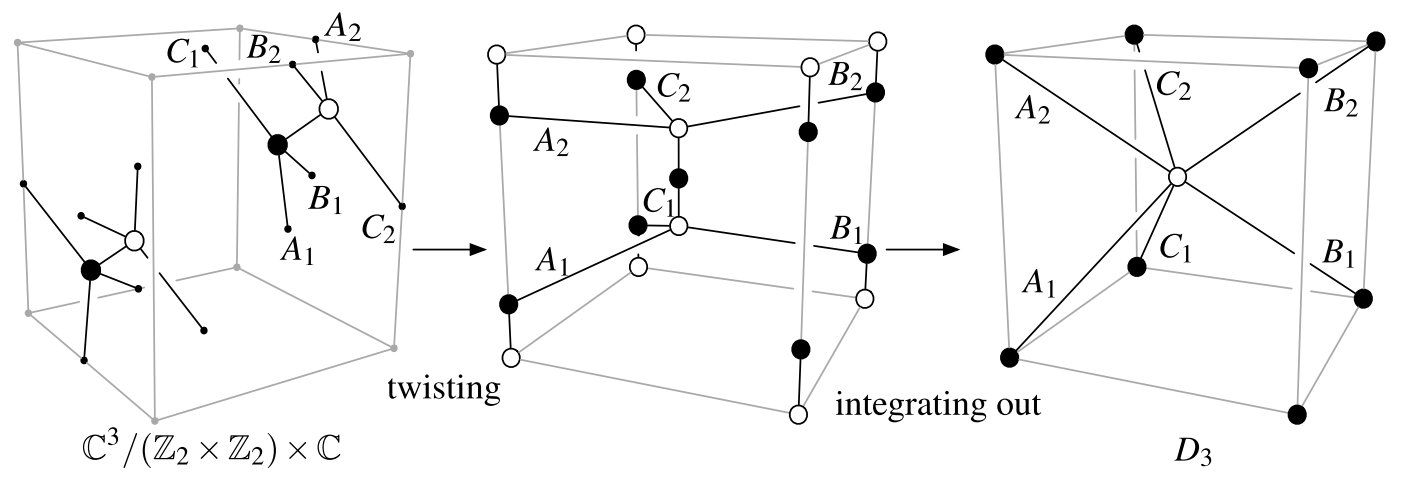

Figure 23: RG flow from $\mathbb{C}^{3} /\left(\mathbb{Z}_{2} \times \mathbb{Z}_{2}\right) \times \mathbb{C}$ to $D_{3}$.

We can summarize the massive deformation in the crystal picture as we did for the KW flow; see Figure 23. Adding the twisted mass term translates to extra twistings on the $\phi_{1,2}$ bonds. Unlike in the KW flow, the lattice vector should be changed in order to keep the new crystal in a unit cell.

The same story holds for our second example, $d P_{3} \times \mathbb{C} \rightarrow C\left(Q^{1,1,1}\right)$. Recall from section 3 that the $d P_{3} \times \mathbb{C}$ theory has the charge assignment, 


\begin{tabular}{c|cccccccc} 
& $\phi_{1}$ & $\phi_{2}$ & $A_{1}$ & $A_{2}$ & $B_{1}$ & $B_{2}$ & $C_{1}$ & $C_{2}$ \\
\hline$Q_{1}$ & 0 & 0 & + & + & - & - & 0 & 0 \\
$Q_{2}$ & 0 & 0 & - & - & 0 & 0 & + & +
\end{tabular}

and the super-potential

$$
W=\phi_{1}\left(A_{1} B_{1} C_{1}-A_{2} B_{2} C_{2}\right)-\phi_{2}\left(A_{1} B_{1} C_{1}-A_{2} B_{2} C_{2}\right) .
$$

Adding the twisted mass term for $\phi_{1}$ and $\phi_{2}$ and integrating them out, we find a vanishing superpotential and the charge matrix of the $C\left(Q^{1,1,1}\right)$ theory. It is also straightforward to show that the algebraic equations describing the moduli space of vacua are related; see examples 1 and 5 in section 4.1. The flow is summarized in Figure 24.

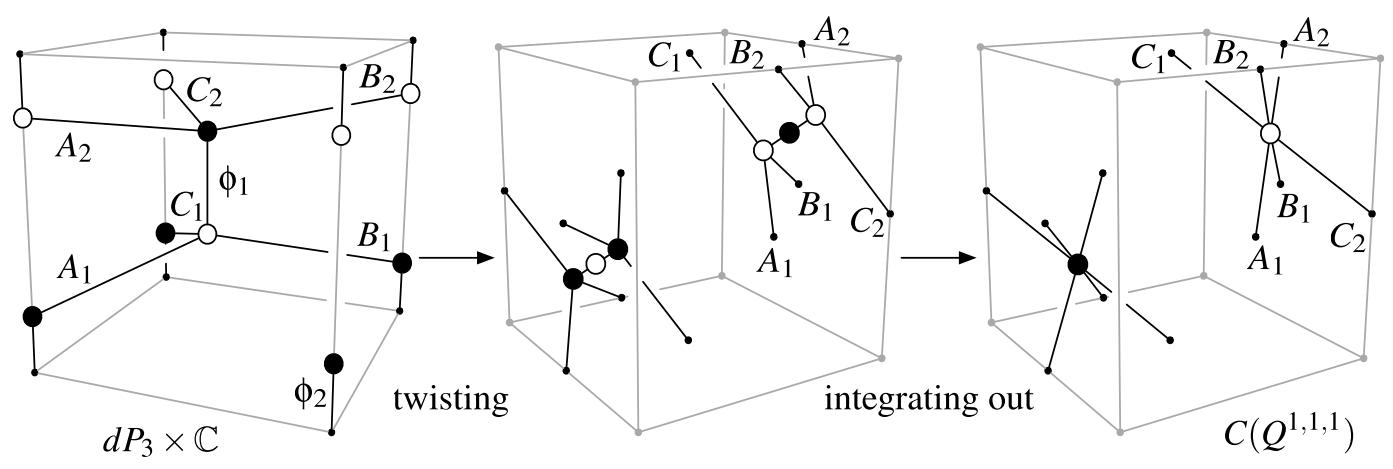

Figure 24: RG flow from $d P_{3} \times \mathbb{C}$ to $C\left(Q^{1,1,1}\right)$.

\section{Discussion}

We made an attempt to write down the world-volume theory of a single M2-brane probes in the context of the crystal model. The resulting effective theory turned out to be an abelian gauge theory. In the crystal model, we identified the gauge groups and found how to read off the charges of the matter fields. The probe theory was shown to have the moduli space of vacua which coincides precisely with the $\mathrm{CY}_{4}$ associated to the crystal. We also discussed toric duality and partial 
resolution of the probe theory. Finally, we found a hint for the existence of new RG flows in M-theory.

One interesting observation is that the geometry probed by M2-brane looks different from that probed by D-branes and fundamental strings. It is known that fundamental string can probe geometric as well as non-geometric phases while D-branes can probe only geometric phases [45, 46]. The study of the abelian gauge theory of M2-brane suggests that the phase structure is more restricted. In the abelian gauge theory, this comes from the less available FI parameters. This also makes the pattern of toric dualities different from that observed in D3-brane theories defined on $\mathrm{CY}_{3}$ singularities. It is an interesting problem to figure out how such restrictions of moduli space arise. In the abelian gauge theory we study, we explored the mesonic branches of the underlying theory and it would be an interesting problem to find the tools to explore the baryonic branches. Recent works [47, 48, 49] may be relevant.

Aside from the ambitious task of constructing the "non-abelian" theory, there are a few directions that deserve further study. First, we hope to find an explicit description of the special Lagrangian manifold $\Sigma$ and the merged world-volume $\mathcal{M}$ discussed in section 2 and 3, so that we can complete the inverse algorithm and verify the behavior of the 2-cycles which give the charges.

Exactly marginal deformations of the $\mathrm{CFT}_{3}$ is another important topic. In $\mathrm{AdS}_{5} / \mathrm{CFT}_{4}$, it is known [50] that a generic toric $\mathrm{CY}_{3}$ with $d$ vertices on the toric diagram admits $(d-1)$ exactly marginal deformations. One of them is the diagonal (complexified) gauge coupling. Another one is the so-called $\beta$-deformation, which attaches phases $e^{ \pm i \pi \beta}$ to the super-potential terms. The other $(d-3)$ deformations in the gauge theory are combinations of relative gauge couplings and super-potential terms. They are interpreted as turning on $B_{N S}+i C_{R R}$ along the $(d-3) 2$-cycles of $Y_{5}$; as shown in [14], $H_{2}\left(Y_{5}, \mathbb{Z}\right)=\mathbb{Z}^{d-3}$, where $Y_{5}$ is the base of the $\mathrm{CY}_{3}$ cone. In the crystal model, there is no analog of the overall gauge coupling. Nor is there deformation due to a vev of a field along homology cycles, because M-theory has only the 3-form field $C$ and $H_{3}\left(Y_{7}, \mathbb{Z}\right)=0$ generically. So, the $\beta$-deformation seems to be the only generic marginal deformation. On the CFT side, it again attaches phases $e^{ \pm i \pi \beta}$ to the super-potential terms. On the supergravity side, it 
corresponds to turning on the $C$-field along the $T^{2} \subset T^{3}$ orthogonal to the $R$-symmetry direction $[51,52,53]$. There is a slight puzzle here. In view of the $D=3, \mathcal{N}=2$ supersymmetry, the parameter $\beta$ is expected to be a complex number, but all the known supergravity solutions have only real values of $\beta$. It will be nice to resolve this puzzle. It will be also interesting to understand non-generic deformations in theories with $\mathcal{N}=4$ or more symmetry using the crystal model and other approaches such as [54].

We conclude the discussion with more speculative comments. Very recently [55], a large class of $\mathrm{CFT}_{3}$ was constructed using Chern-Simons theory coupled to matter fields. It will be interesting to find out whether there is any overlap between this construction and our crystal model. Finally, the $2 \mathrm{~d}$ dimer (tiling) model is known to have many deep connections with other areas of physics such as the Ising model, mirror symmetry of $\mathrm{CY}_{3}$ and black-holes. See [56] and references therein for more information. It is tempting to suspect that similar relation may exist for the $3 \mathrm{~d}$ dimer (crystal) model.

\section{Acknowledgments}

It is our pleasure to thank Hoil Kim and Ho-Ung Yee for useful discussions. Seok Kim is supported in part by the KOSEF Grant R010-2003-000-10391-0. Sangmin Lee is supported by the KOSEF Basic Research Program, Grant R01-2006-000-10965-0. Sungjay Lee is supported in part by the Korea Research Foundation Grant R14-2003-012-01001-0. Jaemo Park is supported by the Science Research Center Program of KOSEF through the Center for Quantum Space-Time (CQUeST) of Sogang University with the grant number R11-2005-021. 


\section{References}

[1] J. M. Maldacena, "The large N limit of superconformal field theories and supergravity," Adv. Theor. Math. Phys. 2, 231(1998) [Int. J. Theor. Phys. 38, 1113 (1999)], arXiv:hepth/9711200.

[2] K. Oh and R. Tatar, "Three dimensional SCFT from M2 branes at conifold singularities," JHEP 9902, 025 (1999), arXiv:hep-th/9810244.

[3] C. Ahn and H. Kim, "Branes at $\mathbb{C}^{4} / \Gamma$ singularity from toric geometry," JHEP 9904, 012 (1999), arXiv:hep-th/9903181.

[4] G. Dall'Agata, " $\mathcal{N}=2$ conformal field theories from M2-branes at conifold singularities," Phys. Lett. B 460, 79 (1999), arXiv:hep-th/9904198.

[5] D. Fabbri, P. Fré, L. Gualtieri, C. Reina, A. Tomasiello, A. Zaffaroni and A. Zampa, “3D superconformal theories from Sasakian seven-manifolds: New nontrivial evidences for $\mathrm{AdS}_{4} / \mathrm{CFT}_{3}$, , Nucl. Phys. B 577, 547 (2000), arXiv:hep-th/9907219.

[6] C. Ahn, " $\mathcal{N}=2$ SCFT and $M$ theory on $\operatorname{AdS}_{4} \times Q(1,1,1)$," Phys. Lett. B 466, 171 (1999), arXiv:hep-th/9908162.

[7] J. P. Gauntlett, D. Martelli, J. Sparks and D. Waldram, "Sasaki-Einstein metrics on $S^{2} \times S^{3}$," Adv. Theor. Math. Phys. 8, 711 (2004), arXiv:hep-th/0403002.

[8] J. P. Gauntlett, D. Martelli, J. F. Sparks and D. Waldram, "A new infinite class of SasakiEinstein manifolds," Adv. Theor. Math. Phys. 8, 987 (2006), arXiv:hep-th/0403038.

[9] M. Cvetic, H. Lu, D. N. Page and C. N. Pope, "New Einstein-Sasaki spaces in five and higher dimensions," Phys. Rev. Lett. 95, 071101 (2005), arXiv:hep-th/0504225.

[10] S. Lee, "Superconformal field theories from crystal lattices," Phys. Rev. D 75, 101901 (2007), arXiv:hep-th/0610204.

[11] S. Lee, S. Lee and J. Park, “Toric $\mathrm{AdS}_{4} / \mathrm{CFT}_{3}$ duals and M-theory crystals,” JHEP 0705, 004 (2007), arXiv:hep-th/0702120. 
[12] A. Hanany and K.D. Kennaway, "Dimer models and toric diagrams," arXiv:hep-th/0503149.

[13] S. Franco, A. Hanany, K. D. Kennaway, D. Vegh and B. Wecht, "Brane dimers and quiver gauge theories," JHEP 0601, 096 (2006), arXiv:hep-th/0504110.

[14] S. Franco, A. Hanany, D. Martelli, J. Sparks, D. Vegh and B. Wecht, "Gauge theories from toric geometry and brane tilings," JHEP 0601, 128 (2006), arXiv:hep-th/0505211.

[15] A. Hanany and D. Vegh, "Quivers, tilings, branes and rhombi," arXiv:hep-th/0511063.

[16] B. Feng, Y. H. He, K. D. Kennaway and C. Vafa, "Dimer models from mirror symmetry and quivering amoebae," arXiv:hep-th/0511287.

[17] Y. Imamura, "Global symmetries and 't Hooft anomalies in brane tilings," JHEP 0612, 041 (2006). arXiv:hep-th/0609163.

[18] M. R. Douglas, B. R. Greene and D. R. Morrison, “Orbifold resolution by D-branes,” Nucl. Phys. B 506, 84 (1997), arXiv:hep-th/9704151.

[19] D. R. Morrison and M. R. Plesser, "Non-spherical horizons. I," Adv. Theor. Math. Phys. 3, 1 (1999), arXiv:hep-th/9810201.

[20] B. Feng, A. Hanany and Y. H. He, "D-brane gauge theories from toric singularities and toric duality,” Nucl. Phys. B 595, 165 (2001), arXiv:hep-th/0003085.

[21] I. R. Klebanov and E. Witten, "Superconformal field theory on threebranes at a Calabi-Yau singularity,” Nucl. Phys. B 536, 199 (1998), arXiv:hep-th/9807080.

[22] D. Martelli, J. Sparks and S. T. Yau, "The geometric dual of $a$-maximisation for toric SasakiEinstein manifolds," Commun. Math. Phys. 268, 39 (2006), arXiv:hep-th/0503183.

[23] D. Martelli, J. Sparks, S. T. Yau, "Sasaki-Einstein manifolds and volume minimisation," arXiv:hep-th/0603021.

[24] S. Benvenuti, B. Feng, A. Hanany and Y. H. He, "Counting BPS operators in gauge theories: Quivers, syzygies and plethystics,” arXiv:hep-th/0608050.

[25] M. Porrati and A. Zaffaroni, "M-theory origin of mirror symmetry in three dimensional gauge theories," Nucl. Phys. B 490, 107 (1997), arXiv:hep-th/9611201. 
[26] M. R. Douglas and G. W. Moore, “D-branes, Quivers, and ALE Instantons,” arXiv:hepth/9603167.

[27] P. B. Kronheimer, "The Construction of ALE spaces as hyperKähler quotients," J. Diff. Geom. 29, 665 (1989).

[28] N. Seiberg and E. Witten, "Gauge dynamics and compactification to three dimensions," arXiv:hep-th/9607163.

[29] K. A. Intriligator and N. Seiberg, "Mirror symmetry in three dimensional gauge theories," Phys. Lett. B 387, 513 (1996), arXiv:hep-th/9607207.

[30] M. Billo, D. Fabbri, P. Fre, P. Merlatti and A. Zaffaroni, "Rings of short $\mathcal{N}=3$ superfields in three dimensions and M-theory on $A d S_{4} \times N(0,1,0)$," Class. Quant. Grav. 18, 1269 (2001), arXiv:hep-th/0005219.

[31] K. M. Lee and H. U. Yee, "New $\mathrm{AdS}_{4} \times X_{7}$ geometries with $\mathcal{N}=6$ in M theory," JHEP 0703, 012 (2007). arXiv:hep-th/0605214.

[32] H. U. Yee, “AdS/CFT with tri-Sasakian manifolds,” arXiv:hep-th/0612002.

[33] I. Garcia-Etxebarria, F. Saad and A. M. Uranga, "Quiver gauge theories at resolved and deformed singularities using dimers," JHEP 0606, 055 (2006), arXiv:hep-th/0603108.

[34] A. Khavaev, K. Pilch and N. P. Warner, "New vacua of gauged $\mathcal{N}=8$ supergravity in five dimensions," Phys. Lett. B 487, 14 (2000), arXiv:hep-th/9812035.

[35] D. Z. Freedman, S. S. Gubser, K. Pilch and N. P. Warner, "Renormalization group flows from holography supersymmetry and a c-theorem," Adv. Theor. Math. Phys. 3, 363 (1999), arXiv:hep-th/9904017.

[36] C. Ahn and S. J. Rey, "Three-dimensional CFTs and RG flow from squashing M2-brane horizon,” Nucl. Phys. B 565, 210 (2000), arXiv:hep-th/9908110.

[37] C. Ahn and S. J. Rey, "More CFTs and RG flows from deforming M2/M5-brane horizon," Nucl. Phys. B 572, 188 (2000), arXiv:hep-th/9911199. 
[38] C. Ahn and J. Paeng, “Three-dimensional SCFTs, supersymmetric domain wall and renormalization group flow,” Nucl. Phys. B 595, 119 (2001), arXiv:hep-th/0008065.

[39] R. Corrado, K. Pilch and N. P. Warner, "An $\mathcal{N}=2$ supersymmetric membrane flow," Nucl. Phys. B 629, 74 (2002), arXiv:hep-th/0107220.

[40] C. Ahn and K. Woo, "Domain wall and membrane flow from other gauged $d=4, \mathcal{N}=8$ supergravity. I,” Nucl. Phys. B 634, 141 (2002), arXiv:hep-th/0109010.

[41] C. Ahn and K. Woo, "Domain wall from gauged $d=4, \mathcal{N}=8$ supergravity. II," JHEP 0311, 014 (2003), arXiv:hep-th/0209128.

[42] D. Nemeschansky and N. P. Warner, "A family of M-theory flows with four supersymmetries," arXiv:hep-th/0403006.

[43] N. Halmagyi, K. Pilch, C. Romelsberger and N. P. Warner, "The complex geometry of holographic flows of quiver gauge theories," JHEP 0609, 063 (2006), arXiv:hep-th/0406147.

[44] S. Kim, S. Lee, S. Lee and J. Park, work in progress.

[45] M. Douglas B. Greene and D. Morrison, Orbifold resolution by D-branes," Nucl. Phys. B 506, 84 (1997), arXiv:hep-th/9704151.

[46] E. Witten, "Phase transitions in M-theory and F-theory," Nucl. Phys. B 471, 195 (1996), arXiv:hep-th/9603150.

[47] A. Butti, D. Forcella and A. Zaffaroni, "Counting BPS baryonic operators in CFTs with Sasaki-Einstein duals," arXiv:hep-th/0611229.

[48] D. Forcella, A. Hanany and A. Zaffaroni, "Baryonic generating functions," arXiv:hepth/0701236.

[49] A. Butti, D. Forcella, A. Hanany, D. Vegh and A. Zaffaroni, "Counting Chiral Operators in Quiver Gauge Theories," arXiv:0705.2771 [hep-th].

[50] Y. Imamura, H. Isono, K. Kimura and M. Yamazaki, "Exactly marginal deformations of quiver gauge theories as seen from brane tilings," arXiv:hep-th/0702049. 
[51] O. Lunin and J. M. Maldacena, "Deforming field theories with $U(1) \times U(1)$ global symmetry and their gravity duals," JHEP 0505, 033 (2005), arXiv:hep-th/0502086.

[52] C. Ahn and J. F. Vazquez-Poritz, "Marginal deformations with $U(1)^{3}$ global symmetry," JHEP 0507, 032 (2005), arXiv:hep-th/0505168.

[53] J. P. Gauntlett, S. Lee, T. Mateos and D. Waldram, "Marginal deformations of field theories with $\mathrm{AdS}_{4}$ duals," JHEP 0508, 030 (2005), arXiv:hep-th/0505207.

[54] B. Kol, “On conformal deformations,” JHEP 0209, 046 (2002), arXiv:hep-th/0205141.

[55] D. Gaiotto and X. Yin, "Notes on superconformal Chern-Simons-matter theories," arXiv:0704.3740 [hep-th].

[56] R. Dijkgraaf, D. Orlando and S. Reffert, "Dimer models, free fermions and super quantum mechanics," arXiv:0705.1645 [hep-th].

[57] S. Lee and S.-J. Rey, "Comments on anomalies and charges of toric-quiver duals," JHEP 0603, 068 (2006), arXiv:hep-th/0601223. 